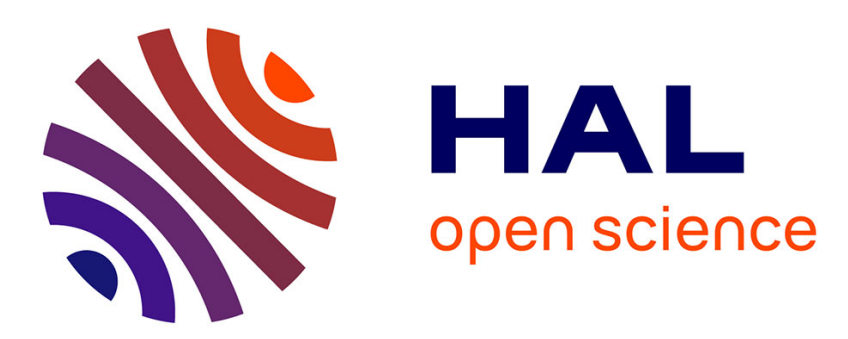

\title{
A wave finite element-based approach for the modeling of periodic structures with local perturbations
}

\author{
J.-M. Mencik, Denis Duhamel
}

\section{To cite this version:}

J.-M. Mencik, Denis Duhamel. A wave finite element-based approach for the modeling of periodic structures with local perturbations. Finite Elements in Analysis and Design, 2016, 121, pp.40 - 51. 10.1016/j.finel.2016.07.010 . hal-01695311

\section{HAL Id: hal-01695311 \\ https://hal.science/hal-01695311}

Submitted on 29 Jan 2018

HAL is a multi-disciplinary open access archive for the deposit and dissemination of scientific research documents, whether they are published or not. The documents may come from teaching and research institutions in France or abroad, or from public or private research centers.
L'archive ouverte pluridisciplinaire HAL, est destinée au dépôt et à la diffusion de documents scientifiques de niveau recherche, publiés ou non, émanant des établissements d'enseignement et de recherche français ou étrangers, des laboratoires publics ou privés. 


\title{
A wave finite element-based approach for the modeling of periodic structures with local perturbations
}

\author{
J.-M. Mencik*,a , D. Duhamel ${ }^{\text {b }}$ \\ ${ }^{a}$ INSA Centre Val de Loire, Université François Rabelais de Tours, LMR EA 2640, Campus de \\ Blois, 3 Rue de la Chocolaterie, CS 23410, 41034 Blois Cedex, France \\ ${ }^{b}$ Université Paris-Est, Laboratoire Navier, ENPC/IFSTTAR/CNRS, 6 et 8 Avenue Blaise Pascal, Cité \\ Descartes, Champs-sur-Marne, 77455 Marne La Vallée Cedex 2, France
}

\begin{abstract}
The wave finite element (WFE) method is investigated to describe the dynamic behavior of finite-length periodic structures with local perturbations. The structures under concern are made up of identical substructures along a certain straight direction, but also contain several perturbed substructures whose material and geometric characteristics undergo arbitrary slight variations. Time-harmonic elasticity is considered. Emphasis is on the development of a numerical tool which is fast and accurate for computing the related forced responses. To achieve this task, a model reduction technique is proposed which involves partitioning a whole periodic structure into one central structure surrounded by two unperturbed substructures, and considering perturbed parts which are composed of perturbed substructures surrounded by two unperturbed ones. In doing so, a few wave modes are only required for modeling the central periodic structure, outside the perturbed parts. For forced response computation purpose, a reduced wave-based matrix formulation is established which follows from the consideration of transfer matrices between the right and left sides of the perturbed parts. Numerical experiments are
\end{abstract}

\footnotetext{
* Corresponding author

Email addresses: jean-mathieu.mencik@insa-cvl.fr(J.-M. Mencik), denis.duhamel@enpc.fr(D. Duhamel)
} 
carried out on a periodic 2D structure with one or two perturbed substructures to validate the proposed approach in comparison with the finite element (FE) method. Also, Monte Carlo (MC) simulations are performed with a view to assessing the sensitivity of a purely periodic structure to the occurrence of arbitrarily located perturbations. A strategy is finally proposed for improving the robustness of periodic structures. It involves artificially adding several "controlled" perturbations for lowering the sensitivity of the dynamic response to the occurrence of other uncontrolled perturbations.

Key words: Wave finite element method, periodic structures, local perturbations, forced response, robust design.

\section{Introduction}

The problem of predicting the dynamic behavior of finite-length periodic structures with local perturbations is tackled in the present paper. Such systems are composed, along a certain straight direction, of identical substructures which can be of arbitrary shape, e.g., like an aircraft fuselage. Assessing the sensitivity of the frequency response of those structures to the occurrence of small perturbations which might be arbitrarily located - e.g., such as variabilities of the design processes, or defects - relates the motivation of the present study. The challenge concerns the development of a numerical approach which is low time-consuming in comparison with the conventional FE method, while keeping the same level of accuracy. Time-harmonic elasticity problems are considered here. Among the wave-based numerical approaches, the WFE method has proved to be relevant for modeling purely periodic structures, and will be thus improved in this work with a view to modeling periodic structures with local perturbations. 
Originally, the WFE method has been developed to describe the wave propagation along one-dimensional periodic structures $[1,2,3]$. The procedure is nothing else but a transfer-matrix approach and the use of the Bloch's theorem [4] for expressing the so-called wave modes, i.e., waves which propagate from substructure to substructure along the right and left directions of a periodic structure. To date, the WFE method has been applied to homogeneous waveguides — such as beams, plates, multi-layered systems and fluid-filled pipes [5, 6, 7, 8, 9] — and simple periodic structures $[10,11]$ or more complex structures such as tires in [12], the cochlea in [13], for the vibroacoustic of thick layered panels in [14] or poroelastic media in [15]. The scattering matrix for guided acoustical propagation has been computed by FEM and WFE in [16]. The method was also used for the computation of metamaterials by [17] and its accuracy was studied for instance by [18]. Besides, the forced response of finite-length periodic structures has been analyzed in various ways by means of the WFE method $[19,20,21,22]$. The strategy consists in expanding vectors of displacements and forces in bases of wave modes. For instance, the study of assemblies involving elastic waveguides and junctions has been proposed in [23], while that of truly periodic structures - i.e., which are made up of heterogeneous substructures — has been investigated in [24]. More recently, a model reduction technique has been proposed for the study of the forced response of periodic structures made up of arbitrary-shaped substructures which are modeled with many degrees of freedom (DOFs) [25]. The strategy consists in partitioning a given periodic structure into one central structure which is modeled by means of the WFE method, and two surrounding substructures which are modeled with the FE method. In doing so, it becomes possible to model the central structure with a small number of wave modes only. This is explained by the fact that the kinematic and mechanical fields admit smooth variations on the boundaries of the central structure - i.e., the interfaces between the central structure and 
the surrounding FE-based substructures. In other words, a small number of wave modes are only needed to accurately capture the boundary conditions (BCs) of the central structure. This strategy has been proved to be relevant, e.g., for computing the forced response of a periodic structure whose substructures are described with more than 30,000 DOFs [25]. The remarkable feature of the approach is that it can be easily implemented on MATLAB ${ }^{\circledR}$, and yields small computational times when compared to dedicated FE softwares.

The dynamic behavior of periodic structures which contain several perturbed substructures - which can be different each other - is analyzed throughout the present paper by means of the WFE method. Within the present scope, those perturbed substructures undergo arbitrary variations of their material and geometric characteristics, and can be located in arbitrary way along a periodic structure. The motivation lies in the development of a numerical tool which is fast and accurate for computing the forced response of these systems. Such an analysis appears to be completely original and has never been carried out previously. Here, original improvements of the reduction technique developed in [25] are proposed which involve introducing perturbed parts, made up of a FE-based perturbed substructure and two surrounding FE-based unperturbed ones. In doing so, one expects smooth variations of the kinematic and mechanical fields on the left and right sides of each perturbed part, i.e., they can be described with a small number of wave modes. In addition, a new reduced wave-based matrix formulation is developed for the computation of the forced response of perturbed periodic structures, and which follows from the consideration of small-sized transfer matrices between the right and left sides of the perturbed parts.

The rest of the paper is organized as follows. In Section 2, the basics of the WFE method are recalled. The numerical strategy used to compute the wave modes which travel along a periodic structure made up of arbitrary-shaped substructures 
is presented. Also, the strategy to compute the forced response of periodic structures is detailed. In Section 3, the forced response of periodic structures with perturbed substructures is analyzed. The aforementioned model reduction technique - which consists in partitioning a whole periodic structure into one central structure surrounded by two FE-based extra substructures, and considering FE-based perturbed parts made up of perturbed substructures and surrounding unperturbed ones - is developed. The derivation of the transfer matrices of the perturbed parts is detailed, along with the wave-based formulation to compute the forced response of periodic structures with local perturbations. In Section 4, numerical experiments are carried out in order to validate the proposed approach in comparison with the conventional FE method. Also, Monte Carlo (MC) simulations are performed to assess the sensitivity of a purely periodic structure to the occurrence of arbitrarily located perturbed substructures. A strategy is finally proposed for improving the robustness of periodic structures. In this framework, several "controlled" perturbed substructures are artificially added for reducing the sensitivity of the dynamic response to the occurrence of other uncontrolled perturbations.

\section{WFE method}

\subsection{Mechanical problem}

The techniques described in this article can be applied to different time-harmonic linear mechanical problems. However, to fix ideas, we consider a two-dimensional plane stress linear elastic problem. We are looking for the displacement $\mathbf{u}(x, y)$ on 
a domain $\Omega$ such that

$$
\operatorname{div} \boldsymbol{\sigma}=-\rho \omega^{2} \mathbf{u} \text { on } \Omega
$$

with $\rho$ the density, $\omega$ the circular frequency, $\sigma$ the stress tensor such that

$$
\sigma=\mathbf{C}: \epsilon
$$

with $\boldsymbol{\epsilon}$ the strain tensor and $\mathbf{C}$ the four dimensional elasticity tensor. In engineering notations, this can also be written as

$$
\left[\begin{array}{c}
\sigma_{x x} \\
\sigma_{y y} \\
\sigma_{x y}
\end{array}\right]=\frac{E}{1-\nu^{2}}\left[\begin{array}{ccc}
1 & \nu & 0 \\
\nu & 1 & 0 \\
0 & 0 & 1-\nu
\end{array}\right]\left[\begin{array}{c}
\epsilon_{x x} \\
\epsilon_{y y} \\
\epsilon_{x y}
\end{array}\right]
$$

with $E$ the Young's modulus and $\nu$ the Poisson's coefficient. The boundary $\Gamma$ is divided into two complementary parts $\Gamma=\Gamma_{u} \cup \Gamma_{t}, \Gamma_{u} \cap \Gamma_{t}=\varnothing$ with

$$
\begin{aligned}
& \mathbf{u}=0 \text { on } \Gamma_{u} \\
& \mathbf{t}=\mathbf{t}_{0} \text { on } \Gamma_{t}
\end{aligned}
$$

with $\mathbf{t}=\boldsymbol{\sigma} . \mathbf{n}$ is the traction vector on the boundary, $\mathbf{n}$ the unit exterior normal vector to the boundary and $\mathbf{t}_{0}$ is a given function. We consider here only homogeneous displacement condition on $\Gamma_{u}$ for simplicity but a non homogeneous displacement condition could be considered with small modifications of the following relations. This problem can be solved by a variational method which consists in multiply the domain equation by a test function $\mathbf{v}(x, y)$ such that $\mathbf{v}=0$ on $\Gamma_{u}$ giving

$$
\int_{\Omega} \mathbf{v} \cdot \operatorname{div} \boldsymbol{\sigma} d x=-\int_{\Omega} \rho \omega^{2} \mathbf{v} \cdot \mathbf{u} d x
$$

integrating by part the first integral yields

$$
-\int_{\Omega} \boldsymbol{\epsilon}(\boldsymbol{v}) \cdot \mathbf{C} \cdot \boldsymbol{\epsilon}(\boldsymbol{u}) d x+\int_{\Gamma_{t}} \mathbf{v} \cdot t_{0} d s=-\int_{\Omega} \rho \omega^{2} \mathbf{v} \cdot \mathbf{u} d x
$$


Then an approximate solution is introduced such that

$$
\begin{aligned}
\mathbf{u}(x, y) & =\sum_{i=1}^{i=n} \mathbf{u}_{i} N_{i}(x, y) \\
\mathbf{v}(x, y) & =\sum_{i=1}^{i=n} \mathbf{v}_{i} N_{i}(x, y)
\end{aligned}
$$

with $N_{i}$ the shape functions. Inserting this in the variational formulation and keeping in mind that it should be true for each value of $\mathbf{v}_{i}$ gives the matrix equations

$$
-\mathbf{K U}+\mathbf{F}=-\omega^{2} \mathbf{M U}
$$

with $\mathbf{K}$ the stiffness matrix, $\mathbf{M}$ the mass matrix and $\mathbf{U}={ }^{T}\left[\mathbf{u}_{1}, \mathbf{u}_{2}, \ldots, \mathbf{u}_{n}\right]$ the vector of displacement at nodes. Introducing the dynamic stiffness matrix $\mathbf{D}=$ $\mathbf{K}-\omega^{2} \mathbf{M}$ and splitting the boundary into left $L$, right $R$ and interior degrees $i$ of freedom as shown in figure (1) leads to the following relation for a period of the structure

$$
\left[\begin{array}{ccc}
\mathbf{D}_{L L} & \mathbf{D}_{L i} & \mathbf{D}_{L R} \\
\mathbf{D}_{i L} & \mathbf{D}_{i i} & \mathbf{D}_{i R} \\
\mathbf{D}_{R L} & \mathbf{D}_{R i} & \mathbf{D}_{R R}
\end{array}\right]\left[\begin{array}{c}
\mathbf{u}_{L} \\
\mathbf{u}_{i} \\
\mathbf{u}_{R}
\end{array}\right]=\left[\begin{array}{c}
\mathbf{F}_{L} \\
0 \\
\mathbf{F}_{R}
\end{array}\right]
$$

It is supposed that there is no force on the interior nodes. Condensing the interior degrees of freedom leads to a relation involving only the boundary degrees of freedom.

$$
\left[\begin{array}{cc}
\mathbf{D}_{L L}^{*} & \mathbf{D}_{L R}^{*} \\
\mathbf{D}_{R L}^{*} & \mathbf{D}_{R R}^{*}
\end{array}\right]\left[\begin{array}{c}
\mathbf{u}_{L} \\
\mathbf{u}_{R}
\end{array}\right]=\left[\begin{array}{c}
\mathbf{F}_{L} \\
\mathbf{F}_{R}
\end{array}\right]
$$

This relation is the starting point of the WFE method described in the following section. One notes that we only need the mass and stiffness matrices of a period. These could be obtained by any finite element software, for instance, by exploiting the powerful modelling capacities of commercial finite element codes. 


\subsection{Wave propagation along periodic structures}

The scope of the present section is to recall the basics of the WFE method for describing waves which travel in right and left directions along a one-dimensional periodic structure, as shown in Figure 1. The structure is composed of identical substructures along a certain straight direction. These are modeled with a same FE mesh, which particularly means that they contain the same number $n$ of DOFs on their left and right boundaries. Also, they are assumed to be linear, elastic and damped by means of a constant loss factor $\eta$.

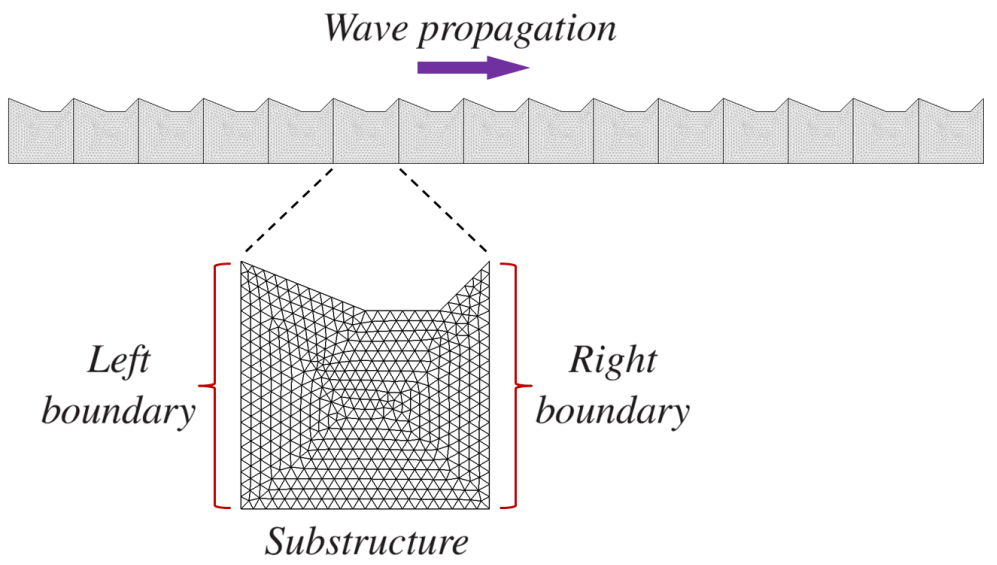

Figure 1: FE mesh of a periodic structure and related substructure.

The first step of the WFE method is to express a relation which links the kinematic/mechanical quantities on the right boundary of a substructure to those on its left boundary. In the frequency domain, it is given by

$$
\left[\begin{array}{c}
\mathbf{q}_{\mathrm{R}} \\
\mathbf{F}_{\mathrm{R}}
\end{array}\right]=\mathbf{S}\left[\begin{array}{c}
\mathrm{q}_{\mathrm{L}} \\
-\mathbf{F}_{\mathrm{L}}
\end{array}\right]
$$

where

$$
\mathbf{S}=\left[\begin{array}{c|c}
-\mathbf{D}_{\mathrm{LR}}^{*-1} \mathbf{D}_{\mathrm{LL}}^{*} & -\mathbf{D}_{\mathrm{LR}}^{*-1} \\
\hline \mathbf{D}_{\mathrm{RL}}^{*}-\mathbf{D}_{\mathrm{RR}}^{*} \mathbf{D}_{\mathrm{LR}}^{*-1} \mathbf{D}_{\mathrm{LL}}^{*} & -\mathbf{D}_{\mathrm{RR}}^{*} \mathbf{D}_{\mathrm{LR}}^{*-1}
\end{array}\right] .
$$


Here, $\mathbf{q}$ and $\mathbf{F}$ are vectors of nodal displacements and nodal forces, respectively, while subscripts $L$ and $R$ denote the DOFs which belong to the left and right boundaries (respectively); also, $\mathbf{D}^{*}$ is the dynamic stiffness matrix (DSM) of the substructure which is condensed on the left and right boundaries [24]. As a second step of the WFE method, the coupling conditions between two consecutive substructures $k$ and $k+1$ are to be considered. If one denotes as $(k+1)$ the interface between the substructures, the coupling conditions are written by $\mathbf{q}_{\mathbf{R}}^{(k+1)}=\mathbf{q}_{\mathbf{L}}^{(k+1)}$ and $\mathbf{F}_{\mathrm{R}}^{(k+1)}=-\mathbf{F}_{\mathrm{L}}^{(k+1)}$. So the displacement is continuous between substructures and there is an equilibrium of forces at the interface between two substructures as we guess that the external forces are put only at the boundary of the structure that is at the first or last interface. As a result of Eqs. (9) and (10), this yields

$$
\left[\begin{array}{c}
\mathbf{q}_{\mathrm{L}}^{(k+1)} \\
-\mathbf{F}_{\mathrm{L}}^{(k+1)}
\end{array}\right]=\mathbf{S}\left[\begin{array}{c}
\mathbf{q}_{\mathrm{L}}^{(k)} \\
-\mathbf{F}_{\mathrm{L}}^{(k)}
\end{array}\right],
$$

where superscript $(k)$ denotes the coupling interface between substructures $k-1$ and $k$. The last step of the WFE method is to compute the eigenvalues $\mu_{j}$ and eigenvectors $\phi_{j}$ of the matrix $\mathbf{S}$ which, according to Bloch's theorem [4], refer to wave parameters and wave shapes. The wave parameters are expressed by $\mu_{j}=$ $\exp \left(-\mathbf{i} \beta_{j} d\right)$, where $d$ is the substructure length while $\beta_{j}$ relates wavenumbers. On the other hand, the wave shapes are expressed in terms of displacement and force vector components - i.e., $\phi_{j}=\left[\phi_{\mathrm{q} j}^{T} \phi_{\mathrm{F} j}^{T}\right]^{T}$ - on each interface between two consecutive substructures. The parameters $\left(\mu_{j}, \phi_{j}\right)$ are referred to as the wave modes of the periodic structure. It is worth emphasizing that the matrix $\mathbf{S}$ in Eq. (10) is symplectic [2], which means that its eigenvalues come in pairs as $\left(\mu_{j}, 1 / \mu_{j}\right)$. This leads to the consideration of $n$ right-going wave modes $\left\{\left(\mu_{j}, \phi_{j}\right)\right\}_{j=1, \ldots, n}$ for which $\left|\mu_{j}\right|<1$, and $n$ left-going wave modes $\left\{\left(\mu_{j}^{\star}, \phi_{j}^{\star}\right)\right\}_{j=1, \ldots, n}$ for which $\mu_{j}^{\star}=1 / \mu_{j}$, with $\left|\mu_{j}^{\star}\right|>1$. In matrix form, those right-going and left-going wave 
modes are written as follows:

$$
\begin{aligned}
& \boldsymbol{\mu}=\left(\boldsymbol{\mu}^{\star}\right)^{-1}=\operatorname{diag}\left\{\mu_{j}\right\}_{j=1, \ldots, n}, \\
& \boldsymbol{\Phi}_{\mathrm{q}}=\left[\phi_{\mathrm{q} 1} \phi_{\mathrm{q} 2} \cdots \phi_{\mathrm{q} n}\right], \quad \boldsymbol{\Phi}_{\mathrm{F}}=\left[\phi_{\mathrm{F} 1} \phi_{\mathrm{F} 2} \cdots \phi_{\mathrm{F} n}\right], \\
& \boldsymbol{\Phi}_{\mathrm{q}}^{\star}=\left[\phi_{\mathrm{q} 1}^{\star} \phi_{\mathrm{q} 2}^{\star} \cdots \phi_{\mathrm{q} n}^{\star}\right], \quad \boldsymbol{\Phi}_{\mathrm{F}}^{\star}=\left[\phi_{\mathrm{F} 1}^{\star} \phi_{\mathrm{F} 2}^{\star} \cdots \phi_{\mathrm{F} n}^{\star}\right],
\end{aligned}
$$

where $\boldsymbol{\Phi}_{\mathrm{q}}, \boldsymbol{\Phi}_{\mathrm{F}}, \boldsymbol{\Phi}_{\mathrm{q}}^{\star}$ and $\boldsymbol{\Phi}_{\mathrm{F}}^{\star}$ are square matrices of size $n \times n$.

\subsection{Wave mode computation}

An efficient strategy has been recently proposed in [25] for computing the wave modes along periodic structures which are made up of arbitrary-shaped substructures. In brief, it makes used of a so-called $\mathbf{S}+\mathbf{S}^{-1}$ transformation [26], which provides an eigenproblem whose eigenvalues are of the form $\lambda_{j}=\mu_{j}+1 / \mu_{j}$, i.e.

$$
\left(\left(\mathbf{N}^{\prime} \mathbf{J L}^{\prime T}+\mathbf{L}^{\prime} \mathbf{J} \mathbf{N}^{\prime} T\right)-\lambda_{j} \mathbf{L}^{\prime} \mathbf{J} \mathbf{L}^{\prime}\right) \mathbf{z}_{j}=\mathbf{0}
$$

where

$$
\mathbf{N}^{\prime} \mathbf{J} \mathbf{L}^{\prime T}+\mathbf{L}^{\prime} \mathbf{J N}^{\prime} T=\left[\begin{array}{cc}
\mathbf{D}_{\mathrm{RL}}^{*}-\mathbf{D}_{\mathrm{LR}}^{*} & \left(\mathbf{D}_{\mathrm{LL}}^{*}+\mathbf{D}_{\mathrm{RR}}^{*}\right) \\
-\left(\mathbf{D}_{\mathrm{LL}}^{*}+\mathbf{D}_{\mathrm{RR}}^{*}\right) & \mathbf{D}_{\mathrm{RL}}^{*}-\mathbf{D}_{\mathrm{LR}}^{*}
\end{array}\right],
$$

and

$$
\mathbf{L}^{\prime} \mathbf{J} \mathbf{L}^{\prime T}=\mathbf{N}^{\prime} \mathbf{J N}^{\prime T}=\left[\begin{array}{cc}
\mathbf{0} & -\mathbf{D}_{\mathrm{RL}}^{*} \\
\mathbf{D}_{\mathrm{LR}}^{*} & \mathbf{0}
\end{array}\right] .
$$

By considering the fact that $\lambda_{j}=\mu_{j}+1 / \mu_{j}$, the wave parameters $\left(\mu_{j}, \mu_{j}^{\star}\right)$ can be found analytically by solving a quadratic equation of the form $x^{2}-\lambda_{j} x+1=0$. Also, the wave shapes are expressed by

$$
\phi_{j}=\left[\begin{array}{cc}
\mathbf{I}_{n} & \mathbf{0} \\
\mathbf{D}_{\mathrm{RR}}^{*} & \mathbf{I}_{n}
\end{array}\right] \mathbf{w}_{j}^{\prime} \quad, \quad \phi_{j}^{\star}=\left[\begin{array}{cc}
\mathbf{I}_{n} & \mathbf{0} \\
\mathbf{D}_{\mathrm{RR}}^{*} & \mathbf{I}_{n}
\end{array}\right] \mathbf{w}_{j}^{\prime},
$$


where $\mathbf{w}_{j}^{\prime}$ and $\mathbf{w}_{j}^{\prime}$ are vectors whose expressions are directly linked to the eigenvectors $\mathbf{z}_{j}$ in Eq. (15), as follows:

$$
\mathbf{w}_{j}^{\prime}=\mathbf{J}\left(\mathbf{L}^{\prime T}-\mu_{j}^{\star} \mathbf{N}^{\prime T}\right) \mathbf{z}_{j} \quad, \quad \mathbf{w}_{j}^{\prime \star}=\mathbf{J}\left(\mathbf{L}^{\prime T}-\mu_{j} \mathbf{N}^{\prime T}\right) \mathbf{z}_{j},
$$

where

$$
\mathbf{J}=\left[\begin{array}{rr}
\mathbf{0} & \mathbf{I}_{n} \\
-\mathbf{I}_{n} & \mathbf{0}
\end{array}\right] .
$$

\subsection{Forced response computation}

The WFE method provides a fast and efficient way to assess the frequency behavior of finite-length periodic structures. The kind of problems which is addressed here can be that of a periodic structure, composed of $N$ substructures, whose left (resp. right) end is subjected to arbitrary vectors of prescribed displacements $\mathbf{q}_{0}$ (resp. $\mathbf{q}_{0}^{\star}$ ) and forces $\mathbf{F}_{0}\left(\right.$ resp. $\mathbf{F}_{0}^{\star}$ ), as shown in Figure 2.

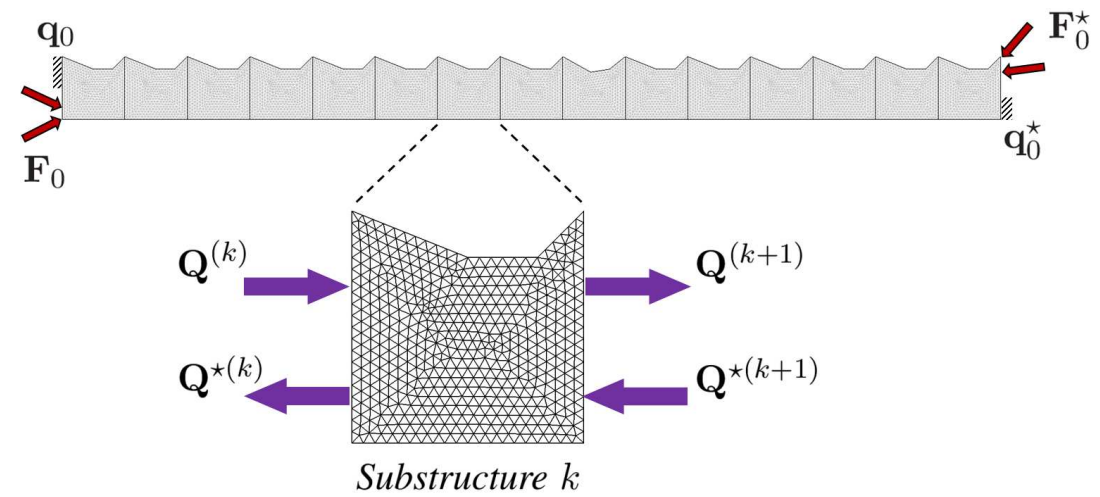

Figure 2: Finite-length periodic structure with prescribed BCs.

The WFE strategy consists in expanding the vectors of displacements and forces, on a given substructure boundary $(k)(k=1, \ldots, N+1)$, in the basis 
of wave shapes, as follows:

$$
\mathbf{q}_{\mathrm{L}}^{(k)}=\mathbf{q}_{\mathrm{R}}^{(k)}=\boldsymbol{\Phi}_{\mathrm{q}} \mathbf{Q}^{(k)}+\boldsymbol{\Phi}_{\mathrm{q}}^{\star} \mathbf{Q}^{\star(k)} \quad, \quad-\mathbf{F}_{\mathrm{L}}^{(k)}=\mathbf{F}_{\mathrm{R}}^{(k)}=\boldsymbol{\Phi}_{\mathrm{F}} \mathbf{Q}^{(k)}+\boldsymbol{\Phi}_{\mathrm{F}}^{\star} \mathbf{Q}^{\star(k)},
$$

where $\mathbf{Q}^{(k)}$ and $\mathbf{Q}^{\star(k)}$ are vectors of wave amplitudes. Also, the spatial variation of the wave amplitudes along the periodic structure is governed by [19]:

$$
\left[\begin{array}{c}
\mathbf{Q}^{(k+1)} \\
\mathbf{Q}^{\star(k+1)}
\end{array}\right]=\mathbb{T}\left[\begin{array}{c}
\mathbf{Q}^{(k)} \\
\mathbf{Q}^{\star(k)}
\end{array}\right] \quad k=1, \ldots, N .
$$

Here, $\mathbb{T}$ is the so-called transfer matrix of a substructure, which is defined by

$$
\mathbb{T}=\left[\begin{array}{cc}
\boldsymbol{\mu} & \mathbf{0} \\
\mathbf{0} & \boldsymbol{\mu}^{-1}
\end{array}\right]
$$

where $\boldsymbol{\mu}$ is the diagonal matrix of the wave parameters that concern the right-going wave modes, see Eq. (12). As a result of Eq. (22), the vectors of wave amplitudes at the left and right ends of the whole structure are linked as $\mathbf{Q}^{(N+1)}=\boldsymbol{\mu}^{N} \mathbf{Q}^{(1)}$ and $\mathbf{Q}^{\star(1)}=\boldsymbol{\mu}^{N} \mathbf{Q}^{\star(N+1)}$. On the other hand, the BCs at the left and right ends need to be expressed in wave-based form, which can be done without difficulty by considering the wave expansion (21) [24]. Regarding for instance vectors of prescribed forces or displacements (Figure 2), the wave-based BCs are given by

$$
\mathbf{Q}^{(1)}=\mathbb{C} \mathbf{Q}^{\star(1)}+\mathbb{F} \quad, \quad \mathbf{Q}^{\star(N+1)}=\mathbb{C}^{\star} \mathbf{Q}^{(N+1)}+\mathbb{F}^{\star},
$$

where $\mathbb{C}$ and $\mathbb{C}^{\star}$ are $n \times n$ scattering matrices whose components relate the reflection coefficients for the wave modes incident to the boundaries, while $\mathbb{F}$ and $\mathbb{F}^{\star}$ are $n \times 1$ vectors which relate the effects of excitation sources. From Eqs. (22) and (24), a whole matrix equation can be established as follows:

$$
\mathcal{A Q}=\mathcal{F}
$$


where

$$
\mathcal{A}=\left[\begin{array}{cc}
\mathbf{I}_{n} & -\mathbb{C} \boldsymbol{\mu}^{N} \\
-\mathbb{C}^{\star} \boldsymbol{\mu}^{N} & \mathbf{I}_{n}
\end{array}\right] \quad, \quad \mathcal{Q}=\left[\begin{array}{c}
\mathbf{Q}^{(1)} \\
\mathbf{Q}^{\star(N+1)}
\end{array}\right] \quad, \quad \mathcal{F}=\left[\begin{array}{c}
\mathbb{F} \\
\mathbb{F}^{\star}
\end{array}\right]
$$

Solving the matrix equation (25) yields the vectors of wave amplitudes $\mathbf{Q}^{(1)}$ and $\mathbf{Q}^{\star(N+1)}$, and by means of Eq. (22) the vectors of wave amplitudes $\mathbf{Q}^{(k)}$ and $\mathbf{Q}^{\star(k)}$ at any substructure boundary $(k)$. Indeed, $\mathbf{Q}^{(k)}=\boldsymbol{\mu}^{k-1} \mathbf{Q}^{(1)}$ and $\mathbf{Q}^{\star(k)}=$ $\boldsymbol{\mu}^{N-(k-1)} \mathbf{Q}^{\star(N+1)}$. Once the vectors of wave amplitudes are computed, the vectors of nodal displacements and forces are simply retrieved by means of Eq. (21). It should be emphasized that the matrix $\mathcal{A}$ in Eq. (26) is likely to be well-conditioned, as explained in $[19,23]$. Also, the size of $\mathcal{A}$ is $2 n \times 2 n-n$ being the number of DOFs on the left or right boundary of a substructure - , i.e., it is small compared to the size of the DSM of the whole periodic structure. As a result, the computation of the inverse of $\mathcal{A}$ can be achieved in a fast and accurate way.

\section{Forced response analysis of periodic structures with local perturbations}

\subsection{Preliminary comments}

The scope of the present study concerns the use of the WFE method for modeling periodic structures with local perturbations. Those perturbations are referred to as some substructures whose geometric or material characteristics are slightly modified compared to the unperturbed ones. For instance, a periodic structure with one perturbed substructure - labeled as $p-$, whose geometric characteristics are slightly modified, is shown in Figure 3.

A first attempt to model such nearly periodic structures with the WFE method would consist in considering the wave-based BCs (24) and expressing transfer 


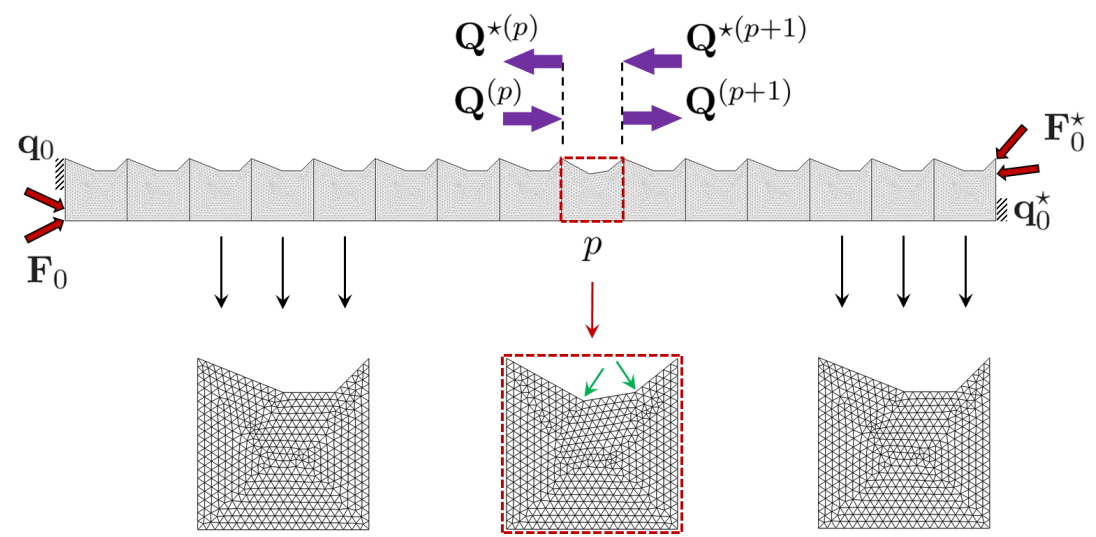

Figure 3: Periodic structure which contains one perturbed substructure $p$ whose geometric characteristics are slightly modified.

matrix-based relations along the unperturbed parts, i.e., outside the perturbed substructures. In this framework, the modeling of each perturbed substructure is carried out by considering a perturbation analysis of the transfer matrix $\mathbb{T}$, see Eq. (22). Unfortunately, this approach is not the right one, which is due to the fact that the matrix $\mathbb{T}$ is highly ill-conditioned. The reason lies in the consideration of both very large and very small components $\mu_{j}$ and $\mu_{j}^{\star}=1 / \mu_{j}$ in $\mathbb{T}$, which are associated to high-order wave modes. In other words, such an approach is subject to high numerical errors, which make it completely inefficient.

Another attempt to analyze the perturbation of the matrix $\mathbb{T}$ would consist in rejecting those high-order wave modes which are source of numerical issues, and then dealing with a reduced wave basis. In doing so, one has to ensure that the reduced wave basis is rich enough to assess the $\mathrm{BCs}$ as well as the right perturbed transfer matrix. Again, things are not so straightforward by considering the fact that some high-order wave modes are usually needed to capture the displacement/force fields, which can be sharp in the vicinity of singularities. In other words, such a reduced basis-based procedure suffers from a lack of accuracy. 
To solve those issues, a strategy is proposed here which is based on the idea to partition a periodic structure into one central structure surrounded by two unperturbed substructures which are modeled in a classical way with the FE method. Also, several perturbed parts are considered which are modeled by means of a FE-based perturbed substructure surrounded by two extra FE-based unperturbed substructures (see Figure 4). In doing so, the displacement/force fields are expected to be smooth on the left and right sides of each perturbed part, as well as on the left and right ends of the central structure, meaning that they can be accurately described with a fairly reduced number of wave modes. This strategy is detailed hereafter.

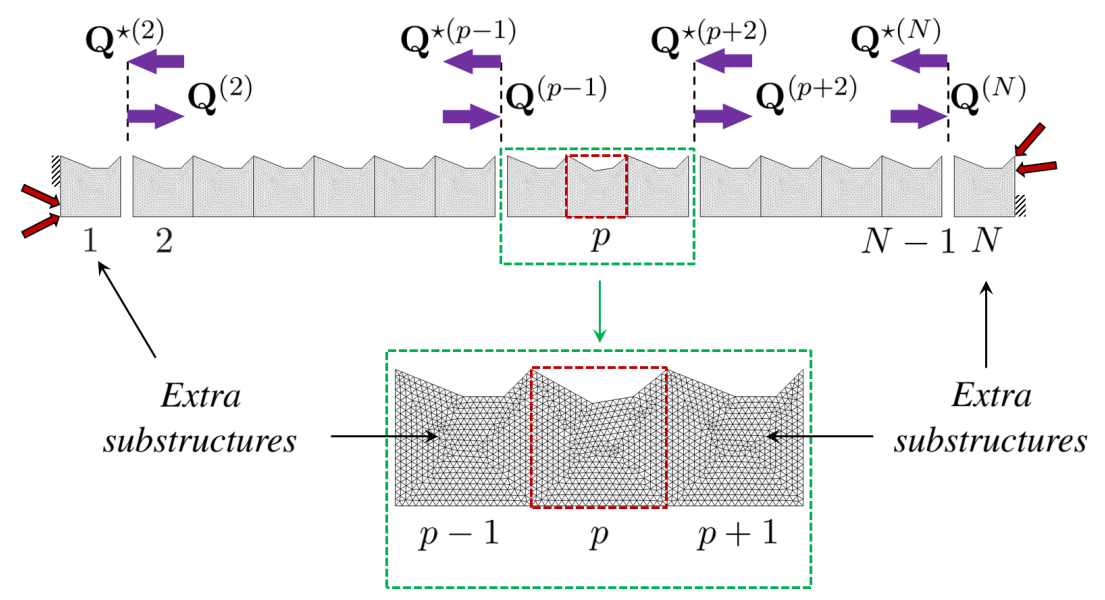

Figure 4: Periodic structure consisting of one central structure (substructures 2 to $\mathrm{N}-1$ ) surrounded by two unperturbed substructures 1 and $\mathrm{N}$, and containing a perturbed part $p$ made up of a perturbed substructure $p$ surrounded by two unperturbed substructures $p-1$ and $p+1$. 


\subsection{Reduced modeling}

Let us model a periodic structure in terms of a central structure, made up of $N-$ 2 substructures, and two extra FE-based unperturbed substructures 1 and $N$ (see Figure 4). The first (1) and last (N) substructures are described by their classical dynamic stiffness matrices obtained from a finite element model. Also, let us model each perturbed part $p$ by means of a FE-based perturbed substructure $p$ surrounded by two FE-based unperturbed substructures $p-1$ and $p+1$. The other substructures are described by the WFE method as it has been shown in [25] that this gives a description of the movement by a limited number of wave modes and leads to a large reductions of the computational cost as long as the considered substructures are far from the boundaries of the structure or from the points of application of the external force. Let us consider reduced $n \times m$ matrices of wave shapes $\widetilde{\boldsymbol{\Phi}}_{\mathrm{q}}=$ $\left[\widetilde{\phi}_{\mathrm{q} 1} \cdots \widetilde{\boldsymbol{\phi}}_{\mathrm{q} m}\right], \widetilde{\boldsymbol{\Phi}}_{\mathrm{F}}=\left[\widetilde{\boldsymbol{\phi}}_{\mathrm{F} 1} \cdots \widetilde{\boldsymbol{\phi}}_{\mathrm{F} m}\right], \widetilde{\boldsymbol{\Phi}}_{\mathrm{q}}^{\star}=\left[\widetilde{\boldsymbol{\phi}}_{\mathrm{q} 1}^{\star} \cdots \widetilde{\phi}_{\mathrm{q} m}^{\star}\right]$ and $\widetilde{\Phi}_{\mathrm{F}}^{\star}=\left[\widetilde{\phi}_{\mathrm{F} 1}^{\star} \cdots \widetilde{\phi}_{\mathrm{F} m}^{\star}\right]$ which concern the first $m$ low-order wave modes among the full sets $\left\{\phi_{j}\right\}_{j=1, \ldots, n}$ and $\left\{\boldsymbol{\phi}_{j}^{\star}\right\}_{j=1, \ldots, n}$, i.e., those associated with the values of $\left|\mu_{j}\right|$ and $\left|\mu_{j}^{\star}\right|$ which are the closest to one.

The selection of those low-order wave modes can be carried out by considering the technique proposed in $[27,25]$. In this framework, an error indicator is considered which invokes error norms of vector and matrix terms. The manner by which this selection strategy can be applied to the present study will be investigated in Section 4. However, for the sake of clarity and conciseness, the derivation of the underlying theoretical content will not be brought here.

By considering reduced matrices of wave shapes, the wave expansion (21) can be rewritten as follows:

$$
\widetilde{\mathbf{q}}_{\mathbf{L}}^{(k)}=\widetilde{\mathbf{q}}_{\mathbf{R}}^{(k)}=\widetilde{\mathbf{\Phi}}_{\mathrm{q}} \widetilde{\mathbf{Q}}^{(k)}+\widetilde{\mathbf{\Phi}}_{\mathbf{q}}^{\star} \widetilde{\mathbf{Q}}^{\star(k)} \quad, \quad-\widetilde{\mathbf{F}}_{\mathrm{L}}^{(k)}=\widetilde{\mathbf{F}}_{\mathrm{R}}^{(k)}=\widetilde{\mathbf{\Phi}}_{\mathrm{F}} \widetilde{\mathbf{Q}}^{(k)}+\widetilde{\mathbf{\Phi}}_{\mathrm{F}}^{\star} \widetilde{\mathbf{Q}}^{\star(k)} .
$$


Let us denote as $\widetilde{\boldsymbol{\mu}}$ the $m \times m$ diagonal matrix of wave parameters $\widetilde{\mu}_{j}$, i.e., $\widetilde{\boldsymbol{\mu}}=$ $\operatorname{diag}\left\{\widetilde{\mu}_{j}\right\}_{j=1, \ldots, m}$. Thus the spatial variation of the wave amplitudes, outside the perturbed parts, is given by (see Eq. (22)):

$$
\left[\begin{array}{c}
\widetilde{\mathbf{Q}}^{(k+1)} \\
\widetilde{\mathbf{Q}}^{\star(k+1)}
\end{array}\right]=\widetilde{\mathbb{T}}\left[\begin{array}{c}
\widetilde{\mathbf{Q}}^{(k)} \\
\widetilde{\mathbf{Q}}^{\star(k)}
\end{array}\right],
$$

where

$$
\widetilde{\mathbb{T}}=\left[\begin{array}{cc}
\widetilde{\boldsymbol{\mu}} & \mathbf{0} \\
\mathbf{0} & \widetilde{\boldsymbol{\mu}}^{-1}
\end{array}\right] .
$$

Regarding BCs at the left and right ends of the central structure, these can be expressed in wave-based form as follows:

$$
\widetilde{\mathbf{Q}}^{(2)}=\widetilde{\mathbb{C}} \widetilde{\mathbf{Q}}^{\star(2)}+\widetilde{\mathbb{F}}, \quad \widetilde{\mathbf{Q}}^{\star(N)}=\widetilde{\mathbb{C}}^{\star} \widetilde{\mathbf{Q}}^{(N)}+\widetilde{\mathbb{F}}^{\star} .
$$

Expressions of $\widetilde{\mathbb{C}}, \widetilde{\mathbb{C}}$, $\widetilde{\mathbb{F}}$ and $\widetilde{\mathbb{F}}^{\star}$ follow from the analysis of the coupling conditions between the central structure and the extra-substructures. They are given by [25]:

$$
\begin{aligned}
& \widetilde{\mathbb{C}}=-\left[\mathbb{D} \widetilde{\boldsymbol{\Phi}}_{\mathrm{q}}-\widetilde{\boldsymbol{\Phi}}_{\mathrm{F}}\right]^{+}\left[\mathbb{D} \widetilde{\boldsymbol{\Phi}}_{\mathrm{q}}^{\star}-\widetilde{\boldsymbol{\Phi}}_{\mathrm{F}}^{\star}\right], \quad \widetilde{\mathbb{C}}^{\star}=-\left[\mathbb{D}^{\star} \widetilde{\boldsymbol{\Phi}}_{\mathrm{q}}^{\star}+\widetilde{\boldsymbol{\Phi}}_{\mathrm{F}}^{\star}\right]^{+}\left[\mathbb{D}^{\star} \widetilde{\boldsymbol{\Phi}}_{\mathrm{q}}+\widetilde{\boldsymbol{\Phi}}_{\mathrm{F}}\right], \quad(31) \\
& \widetilde{\mathbb{F}}=-\left[\mathbb{D} \widetilde{\boldsymbol{\Phi}}_{\mathrm{q}}-\widetilde{\boldsymbol{\Phi}}_{\mathrm{F}}\right]^{+}\left[\mathbb{D}_{\mathrm{q}} \mathbf{q}_{0}+\mathbb{D}_{\mathrm{F}} \mathbf{F}_{0}\right], \quad, \quad \widetilde{\mathbb{F}}^{\star}=-\left[\mathbb{D}^{\star} \widetilde{\boldsymbol{\Phi}}_{\mathrm{q}}+\widetilde{\boldsymbol{\Phi}}_{\mathrm{F}}\right]^{+}\left[\mathbb{D}_{\mathrm{q}}^{\star} \mathbf{q}_{0}^{\star}+\mathbb{D}_{\mathrm{F}}^{\star} \mathbf{F}_{0}^{\star}\right],
\end{aligned}
$$

where the superscript + denotes the left pseudo-inverse, while $\mathbf{q}_{0}$ and $\mathbf{F}_{0}$ (resp. $\mathbf{q}_{0}^{\star}$ and $\mathbf{F}_{0}^{\star}$ ) are vectors of prescribed displacements and forces which may be applied to the left (resp. right) end of the whole structure. Also, $\mathbb{D}$ and $\mathbb{D}^{\star}$ stand for the condensed DSMs of the extra substructures, which can be readily obtained from the DSM of a unperturbed substructure, while $\mathbb{D}_{\mathrm{q}}$ and $\mathbb{D}_{\mathrm{q}}^{\star}\left(\right.$ resp. $\mathbb{D}_{\mathrm{F}}$ and $\mathbb{D}_{\mathrm{F}}^{\star}$ ) are matrices which relate the effects of the excitations $\mathbf{q}_{0}$ and $\mathbf{q}_{0}^{\star}\left(\right.$ resp. $\mathbf{F}_{0}$ and $\left.\mathbf{F}_{0}^{\star}\right)$. 
Finally, the relation linking the wave amplitudes between the right and left sides of each perturbed part $p$ is expressed by

$$
\left[\begin{array}{c}
\widetilde{\mathbf{Q}}^{(p+2)} \\
\widetilde{\mathbf{Q}}^{\star(p+2)}
\end{array}\right]=\left(\widetilde{\mathbb{T}}^{3}+\Delta_{p} \widetilde{\mathbb{T}}^{3}\right)\left[\begin{array}{c}
\widetilde{\mathbf{Q}}^{(p-1)} \\
\widetilde{\mathbf{Q}}^{\star(p-1)}
\end{array}\right],
$$

where $\widetilde{\mathbb{T}}^{3}+\Delta_{p} \widetilde{\mathbb{T}}^{3}$ represents the transfer matrix of the perturbed part, while $\widetilde{\mathbb{T}}^{3}$ is given by

$$
\widetilde{\mathbb{T}}^{3}=\left[\begin{array}{cc}
\widetilde{\boldsymbol{\mu}}^{3} & \mathbf{0} \\
\mathbf{0} & \widetilde{\boldsymbol{\mu}}^{-3}
\end{array}\right] .
$$

\subsection{Transfer matrix of the perturbed parts}

Let us denote as $\mathbf{D}_{p}^{\star}$ the condensed DSM of a perturbed part $p$, i.e., which is composed of a perturbed substructure $p$ surrounded by two unperturbed substructures $p-1$ and $p+1$ (see Figure 4). Here, the dynamic condensation (matrix $\left.\mathbf{D}_{p}^{\star}\right)$ is to be understood w.r.t. the left boundary of substructure $p-1$ and the right boundary of substructure $p+1$, and can be easily achieved by assembling the condensed DSMs of those substructures and condensing the interface DOFs on the left and right sides of the assembly. Hence, the dynamic equilibrium equation of the perturbed part is given by

$$
\left[\begin{array}{l}
\widetilde{\mathbf{F}}_{\mathrm{L}}^{(p-1)} \\
\widetilde{\mathbf{F}}_{\mathrm{R}}^{(p+2)}
\end{array}\right]=\mathbf{D}_{p}^{*}\left[\begin{array}{l}
\widetilde{\mathbf{q}}_{\mathrm{L}}^{(p-1)} \\
\widetilde{\mathbf{q}}_{\mathbf{R}}^{(p+2)}
\end{array}\right] .
$$

Besides, by considering the wave expansion (27), one has:

$$
\begin{array}{ccc}
\widetilde{\mathbf{q}}_{\mathrm{L}}^{(p-1)}=\widetilde{\boldsymbol{\Phi}}_{\mathrm{q}} \widetilde{\mathbf{Q}}^{(p-1)}+\widetilde{\boldsymbol{\Phi}}_{\mathrm{q}}^{\star} \widetilde{\mathbf{Q}}^{\star(p-1)}, \quad \widetilde{\mathbf{q}}_{\mathrm{R}}^{(p+2)}=\widetilde{\boldsymbol{\Phi}}_{\mathbf{q}} \widetilde{\mathbf{Q}}^{(p+2)}+\widetilde{\boldsymbol{\Phi}}_{\mathbf{q}}^{\star} \widetilde{\mathbf{Q}}^{\star(p+2)}, \\
-\widetilde{\mathbf{F}}_{\mathrm{L}}^{(p-1)}=\widetilde{\boldsymbol{\Phi}}_{\mathrm{F}} \widetilde{\mathbf{Q}}^{(p-1)}+\widetilde{\boldsymbol{\Phi}}_{\mathbf{F}}^{\star} \widetilde{\mathbf{Q}}^{\star(p-1)}, & \widetilde{\mathbf{F}}_{\mathrm{R}}^{(p+2)}=\widetilde{\boldsymbol{\Phi}}_{\mathrm{F}} \widetilde{\mathbf{Q}}^{(p+2)}+\widetilde{\boldsymbol{\Phi}}_{\mathrm{F}}^{\star} \widetilde{\mathbf{Q}}^{\star(p+2)} .
\end{array}
$$


Thus:

$$
\begin{aligned}
& {\left[\begin{array}{c}
\widetilde{\mathbf{q}}_{\mathrm{L}}^{(p-1)} \\
\widetilde{\mathbf{q}}_{\mathrm{R}}^{(p+2)}
\end{array}\right]=\widetilde{\mathbf{\Psi}}_{\mathrm{q} 1}\left[\begin{array}{c}
\widetilde{\mathbf{Q}}^{(p-1)} \\
\widetilde{\mathbf{Q}}^{\star(p-1)}
\end{array}\right]+\widetilde{\mathbf{\Psi}}_{\mathrm{q} 2}\left[\begin{array}{c}
\widetilde{\mathbf{Q}}^{(p+2)} \\
\widetilde{\mathbf{Q}}^{\star(p+2)}
\end{array}\right],} \\
& {\left[\begin{array}{l}
\widetilde{\mathbf{F}}_{\mathrm{L}}^{(p-1)} \\
\widetilde{\mathbf{F}}_{\mathrm{R}}^{(p+2)}
\end{array}\right]=-\widetilde{\mathbf{\Psi}}_{\mathrm{F} 1}\left[\begin{array}{c}
\widetilde{\mathbf{Q}}^{(p-1)} \\
\widetilde{\mathbf{Q}}^{\star(p-1)}
\end{array}\right]+\widetilde{\mathbf{\Psi}}_{\mathrm{F} 2}\left[\begin{array}{c}
\widetilde{\mathbf{Q}}^{(p+2)} \\
\widetilde{\mathbf{Q}}^{\star(p+2)}
\end{array}\right],}
\end{aligned}
$$

where

$$
\begin{array}{ll}
\widetilde{\boldsymbol{\Psi}}_{\mathrm{q} 1}=\left[\begin{array}{cc}
\widetilde{\boldsymbol{\Phi}}_{\mathrm{q}} & \widetilde{\boldsymbol{\Phi}}_{\mathrm{q}}^{\star} \\
\mathbf{0} & \mathbf{0}
\end{array}\right], \quad \widetilde{\boldsymbol{\Psi}}_{\mathrm{q} 2}=\left[\begin{array}{cc}
\mathbf{0} & \mathbf{0} \\
\widetilde{\boldsymbol{\Phi}}_{\mathrm{q}} & \widetilde{\boldsymbol{\Phi}}_{\mathrm{q}}^{\star}
\end{array}\right], \\
\widetilde{\boldsymbol{\Psi}}_{\mathrm{F} 1}=\left[\begin{array}{cc}
\widetilde{\boldsymbol{\Phi}}_{\mathrm{F}} & \widetilde{\boldsymbol{\Phi}}_{\mathrm{F}}^{\star} \\
\mathbf{0} & \mathbf{0}
\end{array}\right] \quad, \quad \widetilde{\boldsymbol{\Psi}}_{\mathrm{F} 2}=\left[\begin{array}{cc}
\mathbf{0} & \mathbf{0} \\
\widetilde{\boldsymbol{\Phi}}_{\mathrm{F}} & \widetilde{\boldsymbol{\Phi}}_{\mathrm{F}}^{\star}
\end{array}\right] .
\end{array}
$$

By considering the dynamic equilibrium equation, Eq. (35), this yields

$$
\left[\mathbf{D}_{p}^{*} \widetilde{\mathbf{\Psi}}_{\mathrm{q} 2}-\widetilde{\mathbf{\Psi}}_{\mathrm{F} 2}\right]\left[\begin{array}{c}
\widetilde{\mathbf{Q}}^{(p+2)} \\
\widetilde{\mathbf{Q}}^{\star(p+2)}
\end{array}\right]=-\left[\mathbf{D}_{p}^{*} \widetilde{\mathbf{\Psi}}_{\mathrm{q} 1}+\widetilde{\mathbf{\Psi}}_{\mathrm{F} 1}\right]\left[\begin{array}{c}
\widetilde{\mathbf{Q}}^{(p-1)} \\
\widetilde{\mathbf{Q}}^{\star(p-1)}
\end{array}\right] .
$$

As a result, the transfer matrix $\widetilde{\mathbb{T}}^{3}+\Delta_{p} \widetilde{\mathbb{T}}^{3}$ can be expressed as follows:

$$
\widetilde{\mathbb{T}}^{3}+\Delta_{p} \widetilde{\mathbb{T}}^{3}=-\left[\mathbf{D}_{p}^{*} \widetilde{\mathbf{\Psi}}_{\mathrm{q} 2}-\widetilde{\mathbf{\Psi}}_{\mathrm{F} 2}\right]^{+}\left[\mathbf{D}_{p}^{*} \widetilde{\mathbf{\Psi}}_{\mathrm{q} 1}+\widetilde{\mathbf{\Psi}}_{\mathrm{F} 1}\right]
$$

where $\left[\mathbf{D}_{p}^{*} \widetilde{\mathbf{\Psi}}_{\mathrm{q} 2}-\widetilde{\mathbf{\Psi}}_{\mathrm{F} 2}\right]^{+}$is the left pseudo-inverse of $\left[\mathbf{D}_{p}^{*} \widetilde{\mathbf{\Psi}}_{\mathrm{q} 2}-\widetilde{\mathbf{\Psi}}_{\mathrm{F} 2}\right]$.

\subsection{Forced response computation}

Consider a periodic structure composed of $N$ substructures and containing a certain number $P$ of perturbed substructures $p_{i}(i=1, \ldots, P)$. Recall that, within the present framework, the whole structure is assumed to be modeled by means of a central structure surrounded by two unperturbed substructures. Also, each 
perturbed part, labeled as $p_{i}$, in the central structure is assumed to be modeled by means of a perturbed substructure $p_{i}$ surrounded by two unperturbed substructures $p_{i}-1$ and $p_{i}+1$. Notice however that the analysis of two (or more) consecutive perturbed substructures can be undertaken without difficulty by the proposed approach, and would involve surrounding an assembly of perturbed substructures with two extra unperturbed substructures. However, for the sake of clarity, such a case will not be treated here.

By considering Eqs. (28) and (33), a simple relation can be derived which links the vectors of wave amplitudes at any substructure boundary $(k+1)$, inside the central structure and outside the perturbed parts $p_{i}$, to those at the left end of the central structure. It is written by:

$$
\left[\begin{array}{c}
\widetilde{\mathbf{Q}}^{(k+1)} \\
\widetilde{\mathbf{Q}}^{\star(k+1)}
\end{array}\right]=\widetilde{\mathbb{T}}^{(k-1)-p_{u}} \prod_{i=0}^{u-1}\left(\widetilde{\mathbb{T}}^{3}+\Delta_{p_{u-i}} \widetilde{\mathbb{T}}^{3}\right) \widetilde{\mathbb{T}}^{p^{u}-3}\left[\begin{array}{c}
\widetilde{\mathbf{Q}}^{(2)} \\
\widetilde{\mathbf{Q}}^{\star(2)}
\end{array}\right]
$$

where $p_{i}^{u}=p_{u-i}-p_{u-i-1}$ for $i \neq u-1$ and $p_{u-1}^{u}=p_{1}$, while $p_{u}$ refers to the perturbed part which is the nearest to substructure boundary $(k+1)$. In Eq. (44), $\widetilde{T}^{3}+\Delta_{p_{u-i}} \widetilde{T}^{3}$ refers to the transfer matrices of the perturbed parts $p_{u-i}$ $(i=0, \ldots, u-1)$, while $\widetilde{\mathbb{T}}^{(k-1)-p_{u}}$ and $\widetilde{\mathbb{T}}_{i}^{u-3}$ refer to the transfer matrices of the unperturbed parts, i.e., between the perturbed parts. For the sake of clarity, an illustration of those perturbed and unperturbed parts, and related transfer matrices, is brought in Figure 5.

Considering now the relation between the vectors of wave amplitudes at the left and right ends of the central structure, Eq. (44) leads to

$$
\left[\begin{array}{c}
\widetilde{\mathbf{Q}}^{(N)} \\
\widetilde{\mathbf{Q}}^{\star(N)}
\end{array}\right]=(\widetilde{\boldsymbol{\Lambda}}+\Delta \widetilde{\mathbf{\Lambda}})\left[\begin{array}{c}
\widetilde{\mathbf{Q}}^{(2)} \\
\widetilde{\mathbf{Q}}^{\star(2)}
\end{array}\right]
$$




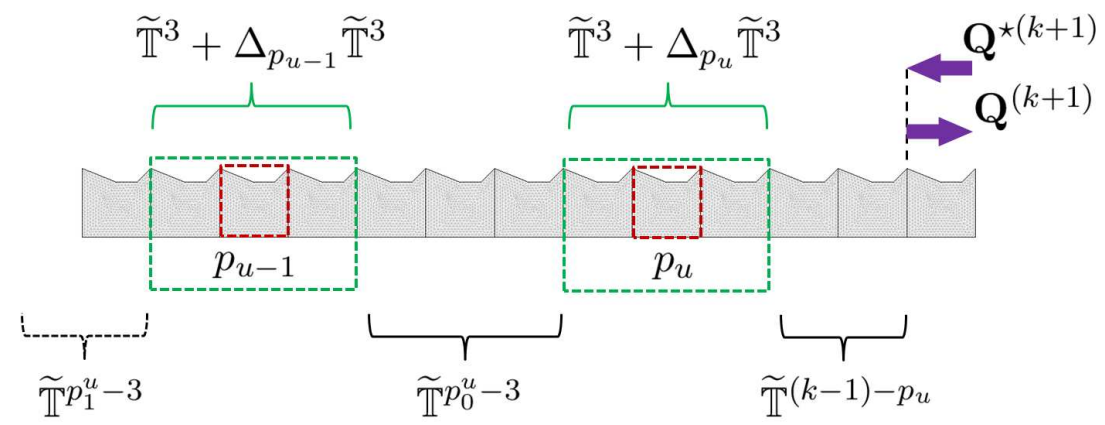

Figure 5: Illustration of the unperturbed and perturbed parts, and related transfer matrices.

where $\widetilde{\Lambda}+\Delta \widetilde{\boldsymbol{\Lambda}}$ is the transfer matrix of the central structure:

$$
\widetilde{\boldsymbol{\Lambda}}+\Delta \widetilde{\boldsymbol{\Lambda}}=\widetilde{\mathbb{T}}^{(N-2)-p_{P}} \prod_{i=0}^{P-1}\left(\widetilde{\mathbb{T}}^{3}+\Delta_{p_{P-i}} \widetilde{\mathbb{T}}^{3}\right) \widetilde{\mathbb{T}}^{p^{P}-3}
$$

Also, $\widetilde{\Lambda}$ refers to the transfer matrix of the unperturbed central structure and is defined by:

$$
\widetilde{\boldsymbol{\Lambda}}=\widetilde{\mathbb{T}}^{N-2}=\left[\begin{array}{cc}
\widetilde{\boldsymbol{\mu}}^{N-2} & \mathbf{0} \\
\mathbf{0} & \widetilde{\boldsymbol{\mu}}^{-(N-2)}
\end{array}\right] .
$$

In addition to Eq. (45), the wave-based BCs at the left and right ends of the central structure need to be considered, see Eq. (30). As a result, a whole matrix equation can be written as follows:

$$
\left[\begin{array}{c|c}
\widetilde{\mathbf{A}} & \widetilde{\mathbf{B}} \\
\hline-(\widetilde{\boldsymbol{\Lambda}}+\Delta \widetilde{\boldsymbol{\Lambda}}) & \mathbf{I}_{2 m}
\end{array}\right]\left[\begin{array}{c}
\widetilde{\mathbf{Q}}^{(2)} \\
\widetilde{\mathbf{Q}}^{\star(2)} \\
\hline \widetilde{\mathbf{Q}}^{(N)} \\
\widetilde{\mathbf{Q}}^{\star(N)}
\end{array}\right]=\left[\begin{array}{c}
\widetilde{\mathbb{F}} \\
\widetilde{\mathbb{F}}^{\star} \\
\hline \mathbf{0} \\
\mathbf{0}
\end{array}\right]
$$

where

$$
\widetilde{\mathbf{A}}=\left[\begin{array}{cc}
\mathbf{I}_{m} & -\widetilde{\mathbb{C}} \\
\mathbf{0} & \mathbf{0}
\end{array}\right] \quad, \quad \widetilde{\mathbf{B}}=\left[\begin{array}{cc}
\mathbf{0} & \mathbf{0} \\
-\widetilde{\mathbb{C}}^{\star} & \mathbf{I}_{m}
\end{array}\right] .
$$


By condensing the matrix equation (48) w.r.t. the first row block, this gives

$$
[\widetilde{\mathbf{A}}+\widetilde{\mathbf{B}}(\widetilde{\boldsymbol{\Lambda}}+\Delta \widetilde{\boldsymbol{\Lambda}})]\left[\begin{array}{c}
\widetilde{\mathbf{Q}}^{(2)} \\
\widetilde{\mathbf{Q}}^{\star(2)}
\end{array}\right]=\left[\begin{array}{c}
\widetilde{\mathbb{F}} \\
\widetilde{\mathbb{F}}^{\star}
\end{array}\right]
$$

It is worth emphasizing that the inversion of the matrix on the left-hand side of Eq. (50) is prone to numerical ill-conditioning, which is due to the occurrence of matrix terms $\widetilde{\boldsymbol{\mu}}^{N-2}$ and $\widetilde{\boldsymbol{\mu}}^{-(N-2)}$ in $\widetilde{\boldsymbol{\Lambda}}$. To solve this issue, the following preconditioner is considered:

$$
\widetilde{\boldsymbol{\Gamma}}=\left[\begin{array}{cc}
\mathbf{I}_{m} & \mathbf{0} \\
\mathbf{0} & \widetilde{\boldsymbol{\mu}}^{N-2}
\end{array}\right] .
$$

A simple proof which highlights the relevance of using such a preconditioner is to consider the matrix $\widetilde{\mathbf{A}}+\widetilde{\mathbf{B}} \widetilde{\boldsymbol{\Lambda}}$, which is related to the unperturbed central structure. By right-multiplying $\widetilde{\mathbf{A}}+\widetilde{\mathbf{B}} \widetilde{\mathbf{\Lambda}}$ by $\widetilde{\boldsymbol{\Gamma}}$, this gives $[\widetilde{\mathbf{A}}+\widetilde{\mathbf{B}} \widetilde{\mathbf{\Lambda}}] \widetilde{\boldsymbol{\Gamma}}$ :

$$
[\widetilde{\mathbf{A}}+\widetilde{\mathbf{B}} \widetilde{\boldsymbol{\Lambda}}] \widetilde{\boldsymbol{\Gamma}}=\left[\begin{array}{cc}
\mathbf{I}_{m} & -\widetilde{\mathbb{C}} \widetilde{\boldsymbol{\mu}}^{N-2} \\
-\widetilde{\mathbb{C}}^{\star} \widetilde{\boldsymbol{\mu}}^{N-2} & \mathbf{I}_{m}
\end{array}\right]
$$

In contrast, $\widetilde{\mathbf{A}}+\widetilde{\mathbf{B}} \widetilde{\mathbf{\Lambda}}$ is given by:

$$
\widetilde{\mathbf{A}}+\widetilde{\mathbf{B}} \widetilde{\boldsymbol{\Lambda}}=\left[\begin{array}{cc}
\mathbf{I}_{m} & -\widetilde{\mathbb{C}} \\
-\widetilde{\mathbb{C}}^{\star} \widetilde{\boldsymbol{\mu}}^{N-2} & \widetilde{\boldsymbol{\mu}}^{-(N-2)}
\end{array}\right] .
$$

The interesting feature of $[\widetilde{\mathbf{A}}+\widetilde{\mathbf{B}} \widetilde{\mathbf{\Lambda}}] \widetilde{\boldsymbol{\Gamma}}$ is that (i) it is composed of diagonal block terms which are identity matrices, and (ii) it contains off-diagonal block terms of the form $\widetilde{\boldsymbol{\mu}}^{N-2}$, with the property that $\|\widetilde{\boldsymbol{\mu}}\|_{2}<1$. As discussed in [19], this makes $[\widetilde{\mathbf{A}}+\widetilde{\mathbf{B}} \widetilde{\boldsymbol{\Lambda}}] \widetilde{\boldsymbol{\Gamma}}$ well-conditioned compared to $\widetilde{\mathbf{A}}+\widetilde{\mathbf{B}} \widetilde{\boldsymbol{\Lambda}}$, where for instance both terms $\widetilde{\boldsymbol{\mu}}^{N-2}$ and $\widetilde{\boldsymbol{\mu}}^{-(N-2)}$ occur.

Hence, by considering the preconditioner $\widetilde{\Gamma}$, Eq. (50) can be rewritten as

$$
([\widetilde{\mathbf{A}}+\widetilde{\mathbf{B}}(\widetilde{\boldsymbol{\Lambda}}+\Delta \widetilde{\mathbf{\Lambda}})] \widetilde{\boldsymbol{\Gamma}}) \widetilde{\boldsymbol{\Gamma}}^{-1}\left[\begin{array}{c}
\widetilde{\mathbf{Q}}^{(2)} \\
\widetilde{\mathbf{Q}}^{\star(2)}
\end{array}\right]=\left[\begin{array}{c}
\widetilde{\mathbb{F}} \\
\widetilde{\mathbb{F}}^{\star}
\end{array}\right]
$$


where

$$
\widetilde{\boldsymbol{\Gamma}}^{-1}=\left[\begin{array}{cc}
\mathbf{I}_{m} & \mathbf{0} \\
\mathbf{0} & \widetilde{\boldsymbol{\mu}}^{-(N-2)}
\end{array}\right] .
$$

Solving Eq. (54) provides the vectors of wave amplitudes at the left end of the central structure, as follows:

$$
\left[\begin{array}{c}
\widetilde{\mathbf{Q}}^{(2)} \\
\widetilde{\mathbf{Q}}^{\star(2)}
\end{array}\right]=\widetilde{\boldsymbol{\Gamma}}([\widetilde{\mathbf{A}}+\widetilde{\mathbf{B}}(\widetilde{\boldsymbol{\Lambda}}+\Delta \widetilde{\boldsymbol{\Lambda}})] \widetilde{\boldsymbol{\Gamma}})^{-1}\left[\begin{array}{c}
\widetilde{\mathbb{F}} \\
\widetilde{\mathbb{F}}^{\star}
\end{array}\right],
$$

which ultimately provides the vectors of wave amplitudes at any interface between the unperturbed substructures, see Eq. (44). In addition, the vectors of nodal displacements and forces on the substructure interfaces can be easily retrieved from Eq. (27).

Remark. The proposed strategy enables one to model periodic structures with several arbitrary perturbed substructures. Notice that any simplified expression based on a first- or second-order Taylor approximation of the transfer matrix (44), about $\widetilde{\mathbb{T}}^{3}$, may not be relevant here. The reason lies in the fact that $\left\|\Delta \widetilde{\mathbb{T}}^{3}\right\|(\|\cdot\|$ being for instance the Frobenius norm) is large compared to $\left\|\widetilde{T}^{3}\right\|$, because the wave modes are strongly coupled to each other as soon as the frequency exceeds a certain threshold. Such a wave mode coupling partly results from the wave reflection mechanisms which occur around each perturbed substructure. The wave modes propagates freely and independently in periodic structures. But around the perturbed substructure, the structure is no longer periodic and an incident wave mode generates both reflected other wave modes and transmitted other wave modes as would do usual waves at an interface between two different media. Also, although the material and geometric characteristics of the substructures are supposed to be slightly modified, the resulting perturbations of the DSMs - i.e., $\left\|\mathbf{D}_{p}^{*}-\mathbf{D}^{*}\right\|-$ are not necessarily small compared to $\left\|\mathbf{D}^{*}\right\|$. 


\subsection{Discussion}

Eq. (56) provides a simple way to quantify how much the dynamic behavior of a periodic structure is sensitive to the occurrence of a certain number of perturbed substructures. In Eq. (56), a matrix is to be inverted which is expressed as $[\widetilde{\mathbf{A}}+$ $\widetilde{\mathbf{B}} \widetilde{\boldsymbol{\Lambda}}] \widetilde{\boldsymbol{\Gamma}}+\widetilde{\mathbf{B}}(\Delta \widetilde{\boldsymbol{\Lambda}}) \widetilde{\boldsymbol{\Gamma}}$, where $[\widetilde{\mathbf{A}}+\widetilde{\mathbf{B}} \widetilde{\boldsymbol{\Lambda}}] \widetilde{\boldsymbol{\Gamma}}$ is given by Eq. (52), while $\widetilde{\mathbf{B}}(\Delta \widetilde{\boldsymbol{\Lambda}}) \widetilde{\boldsymbol{\Gamma}}$ plays the role of the perturbation. Here, $\Delta \widetilde{\boldsymbol{\Lambda}}$ is expressed by (cf. Eqs. (46) and (47)):

$$
\Delta \widetilde{\boldsymbol{\Lambda}}=(\widetilde{\boldsymbol{\Lambda}}+\Delta \widetilde{\boldsymbol{\Lambda}})-\widetilde{\boldsymbol{\Lambda}}=\widetilde{\mathbb{T}}^{(N-2)-p_{P}} \prod_{i=0}^{P-1}\left(\widetilde{\mathbb{T}}^{3}+\Delta_{p_{P-i}} \widetilde{\mathbb{T}}^{3}\right) \widetilde{\mathbb{T}}^{p_{i}^{P}-3}-\widetilde{\mathbb{T}}^{N-2} .
$$

For instance, a simple optimization procedure can be carried out so as to identify the substructure locations $p_{i}$ which minimize the perturbation $\|\widetilde{\mathbf{B}}(\Delta \widetilde{\mathbf{\Lambda}}) \widetilde{\boldsymbol{\Gamma}}\|$ over some frequency bands. This leads the way to the design of weakly sensitive structures which, as such, may be modeled as purely periodic.

Another interesting challenge concerns the robust design of periodic structures by artificially adding several perturbed substructures. In this sense, one seeks to reduce the dispersion of the frequency response functions (FRFs) by introducing several "controlled" perturbations, at locations where a purely periodic structure is highly sensitive to the occurrence of other perturbations which are not controlled (e.g., such as defects). Such an analysis will be carried out in the next section. 


\section{Numerical results}

\subsection{Validation of the WFE modeling}

Consider a periodic 2D structure as shown in Figure 2, which is made up of $N=15$ substructures whose characteristics are: density of $7800 \mathrm{~kg} / \mathrm{m}^{3}$, Young's modulus of $210 G P a$, Poisson ratio of 0.3 , loss factor of 0.005 , length of $0.1 \mathrm{~m}$, height of $0.1 \mathrm{~m}$ and thickness of $0.001 \mathrm{~m}$. The whole structure is clamped on its right end, and it is subjected to a longitudinal point force on the left end. The upper and lower boundaries are free. The substructures are meshed in the same way using 2D plane stress linear triangles, with two DOFs per node, leading to 1024 DOFs for each substructure and $n=42$ DOFs over each left/right boundary. Two test cases are considered here, i.e.

- The structure contains one perturbed substructure located at $p=9$ and whose geometric characteristics are slightly perturbed, as shown in Figure 6.

- The structure contains two perturbed substructures located at $p_{1}=10$ and $p_{2}=13$, as shown in Figure 7. In this case, the geometric characteristics of both substructures are modified in the same way. However, the loss factor of substructure $p_{2}=13$ is perturbed so that $\eta=0.008$, rather than 0.005 for substructure $p_{1}=10$ and the rest of the periodic structure.

For each test case, the FRF - i.e., the frequency evolution of the magnitude of the longitudinal displacement at the excitation point — is assessed at 1000 discrete frequencies which are uniformly spread on a frequency band $[5 \mathrm{~Hz}, 5000 \mathrm{~Hz}]$. The computation of the FRF is achieved by solving Eq. (56) at each discrete frequency. Assessing the vectors of displacements follows from Eqs. (44) and (27). Within the present framework, only a reduced set of wave modes is considered, which in 


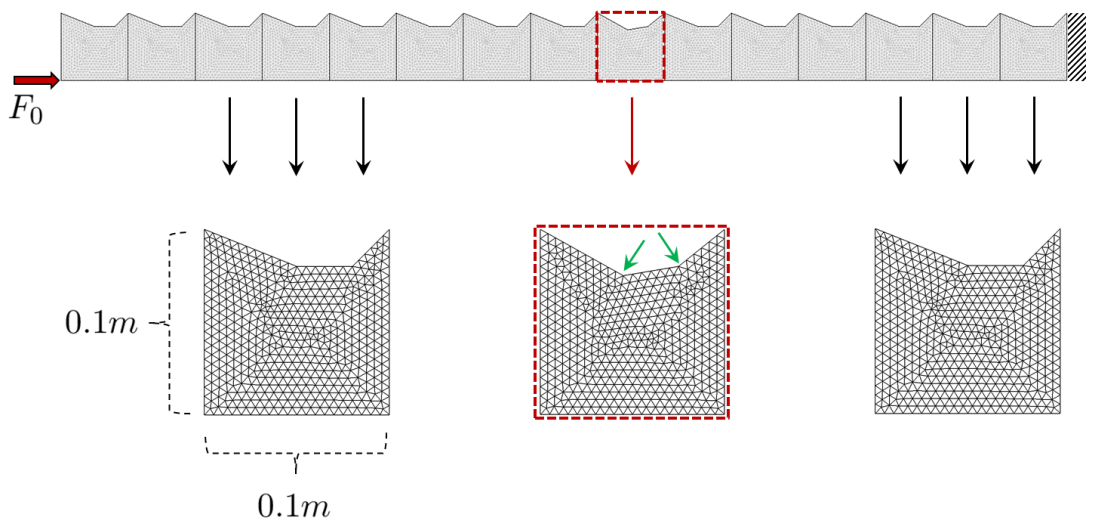

Figure 6: Periodic structure consisting of $N=15$ substructures and containing one perturbed substructure $p=9$.

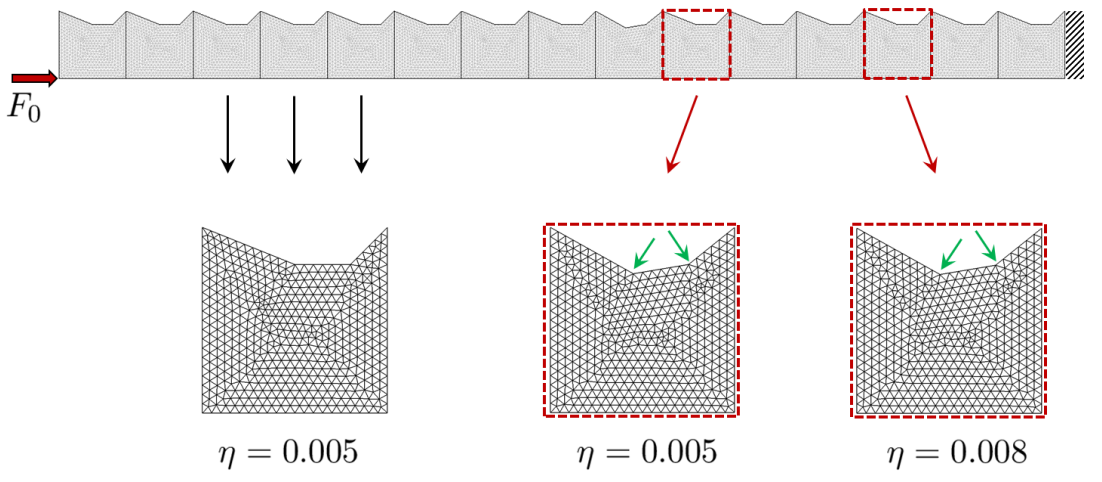

Figure 7: Periodic structure consisting of $N=15$ substructures and containing two perturbed substructures $p_{1}=10$ and $p_{2}=13$.

the present application consists of $m=3$ right/left-going wave modes. The choice of this number $m=3$ results from the model reduction technique proposed in [25]. In brief, it consists in neglecting the strongly evanescent wave modes, i.e., those for which the values of $\left|\mu_{j}\right|$ (resp. $\left|\mu_{j}^{\star}\right|$ ) are too small (resp. too large) so that their decaying rate is strong across substructures.

For comparison purpose, reference FE-based FRFs issued from a commercial 
FE software are also calculated, regarding the perturbed and unperturbed periodic structures. The results are shown in Figures 8 and 9. As it can be seen, the WFEbased FRFs completely match the FE-based solutions over the whole frequency band. In fact, the efficiency of the WFE method lies in the reduction of the computational times. Clearly, it takes less than $40 s$ with the WFE method to compute each FRF using MATLAB ${ }^{\circledR}$ and an Intel ${ }^{\circledR}$ Core $^{\text {TM }}$ i7-3720QM processor, against $280 s$ with a FE software and the same processor. This means $85 \%$ time saving in benefit of the proposed approach. This remarkable feature of the WFE strategy lies in the consideration of a very small matrix system, Eq. (54), which in the present case is of size $6 \times 6$. As it stands, the proposed approach hence constitutes a fast and accurate numerical tool which can be advantageously used to assess e.g. the sensitivity of periodic structures to local perturbations.
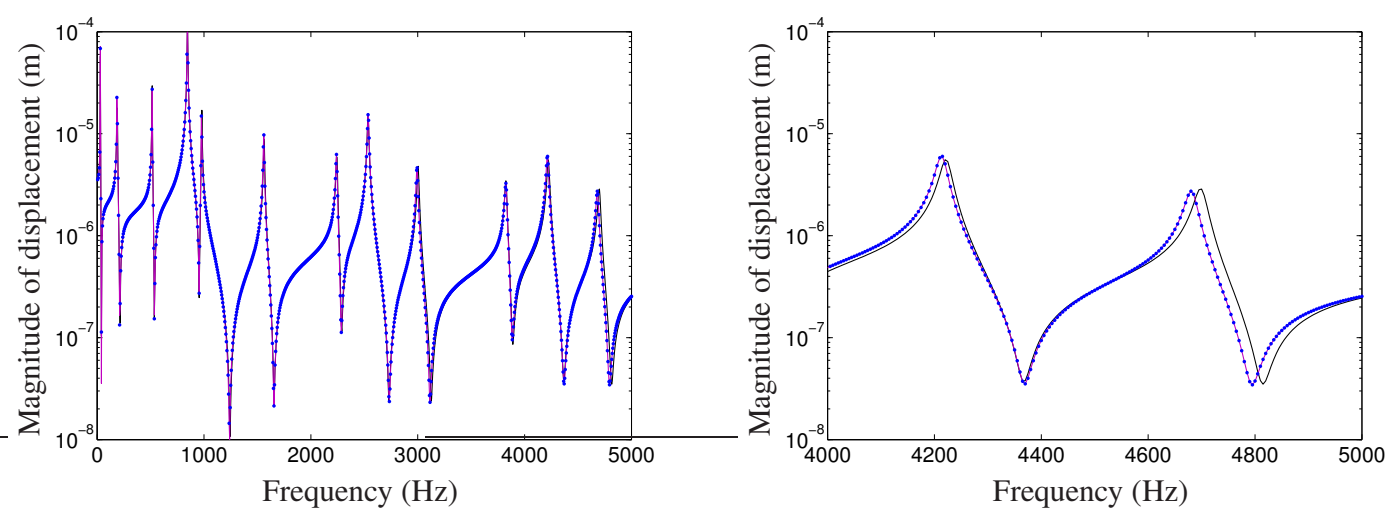

Figure 8: FRF of the periodic structure with one perturbed substructure $p=9$ : FE solution (violet solid line); WFE solution (blue dotted line). FE-based FRF of the unperturbed structure (black solid line). 

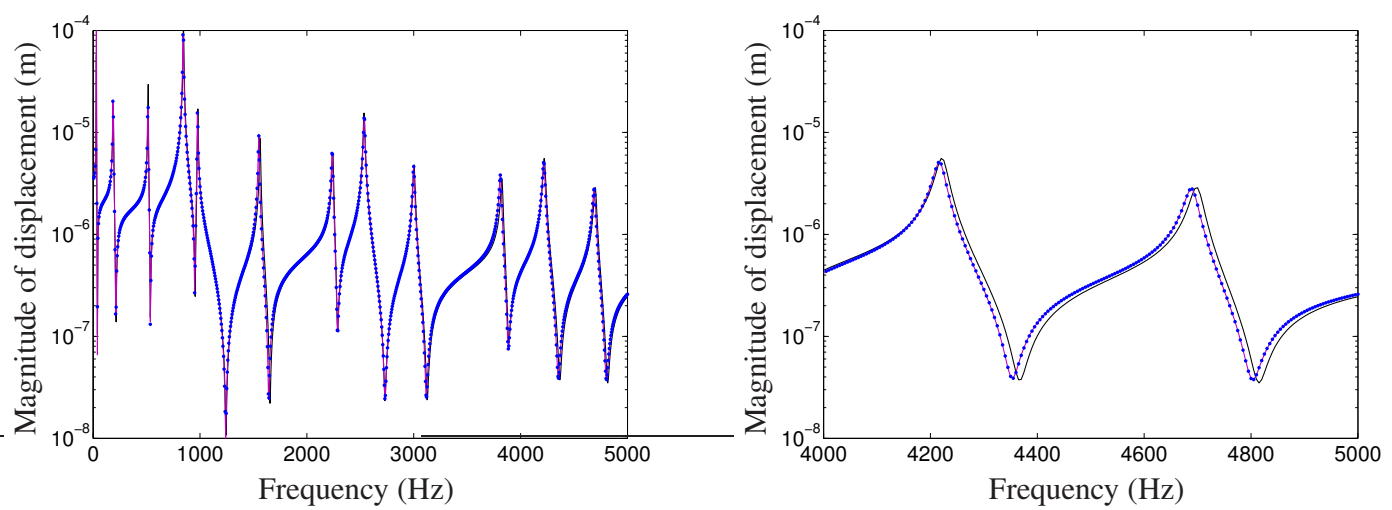

Figure 9: FRF of the periodic structure with two perturbed substructures $p_{1}=10$ and $p_{2}=13$ : FE solution (violet solid line); WFE solution (blue dotted line). FE-based FRF of the unperturbed structure (black solid line).

\subsection{Sensitivity analysis}

The question may arise how much the FRF of a purely periodic structure is sensitive to small perturbations which are known, but whose locations along the structure are uncertain. The proposed approach can be used efficiently to address this task in performing Monte Carlo (MC) simulations at a low computational cost. Two test cases are considered here, which respectively concern (i) one arbitrarily-located perturbed substructure like the one displayed in Figure 6, and (ii) two arbitrarily-located perturbed substructures which are identical to the one shown in Figure 6. The related results are displayed in Figures 10 and 11. It is worth recalling that, within the present framework, each perturbed substructure is surrounded by two unperturbed substructures, which particularly means that two perturbed substructures are at least separated by two unperturbed ones.

Regarding Figures 10 and 11, it is seen that the FRF of the periodic structure becomes more sensitive as the number of perturbed substructures increases and the frequency grows. Such tendencies are predicted, here, in a very fast way by means of the WFE approach, i.e., less than $40 s$ with MATLAB ${ }^{\circledR}$ for each case. Notice 
that this value remains of the same order as for the analysis of the periodic structure with fixed perturbations, which is explained because the wave modes do not need to be recomputed at each MC iteration. In comparison, the FE method would have required more than $3000 s$, which fully gives credit to the proposed approach.
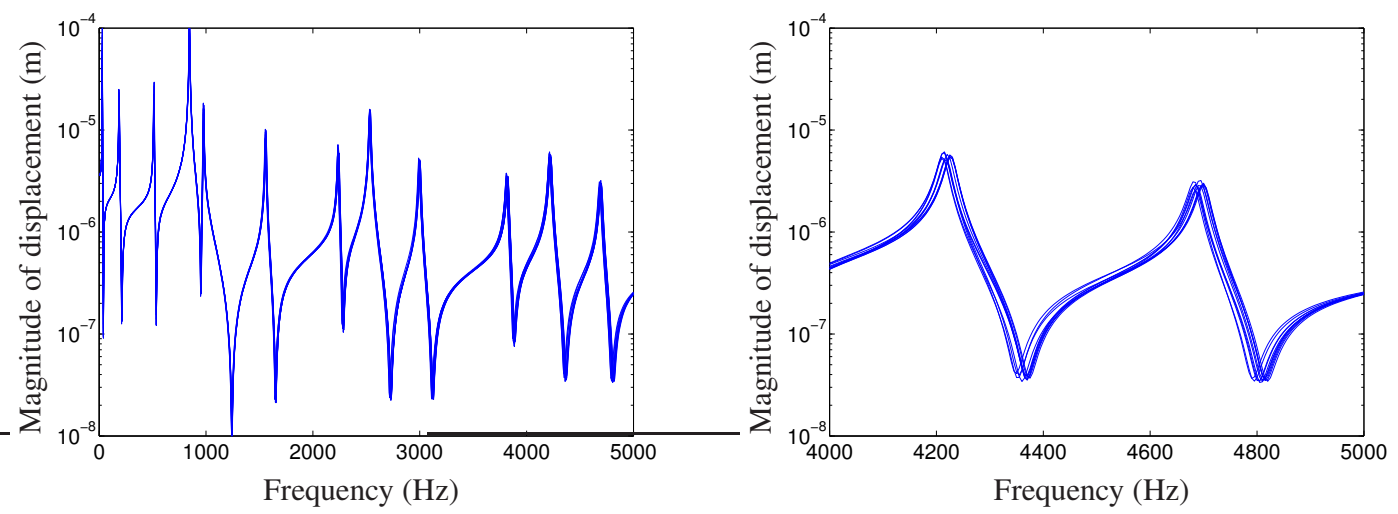

Figure 10: FRF of the periodic structure with one perturbed substructure whose location arbitrarily varies between $p=3$ and $p=13$.
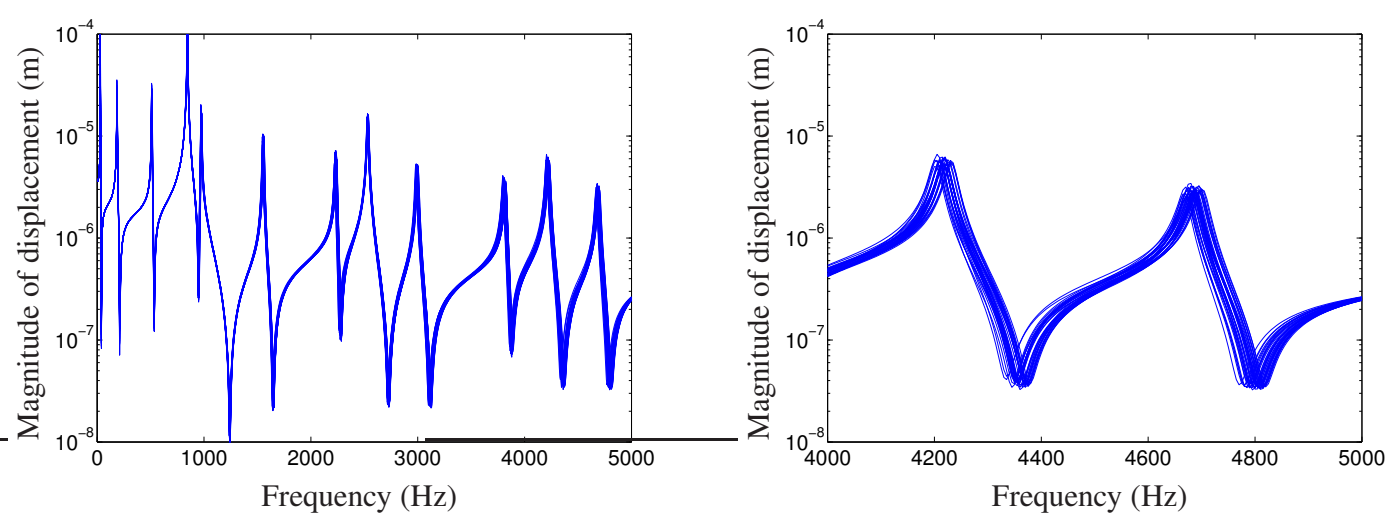

Figure 11: FRF of the periodic structure with two perturbed substructures whose locations arbitrarily vary between $p=3$ and $p=13$. 


\subsection{Robust design}

Consider a periodic structure with $N=30$ substructures like those previously described, and which contains one perturbed substructure whose location along the structure arbitrarily varies. This substructure is perturbed in the sense that its DSM is expressed by $\mathbf{D}^{*}+\varepsilon \mathbf{D}^{*}$, where $\mathbf{D}^{*}$ is the DSM of the unperturbed substructures and $\varepsilon$ is a small parameter, say $\varepsilon=5 \%$. In addition, two so-called "controlled" perturbed substructures are considered, whose shapes are similar to those of the previously studied perturbed substructures (see Figure 12). These substructures are supposed to be significantly perturbed in the sense that their DSM is $0.6 \times \mathbf{D}_{p}^{*}$, where $\mathbf{D}_{p}^{*}$ is the DSM of the perturbed substructures depicted in the previous subsection.

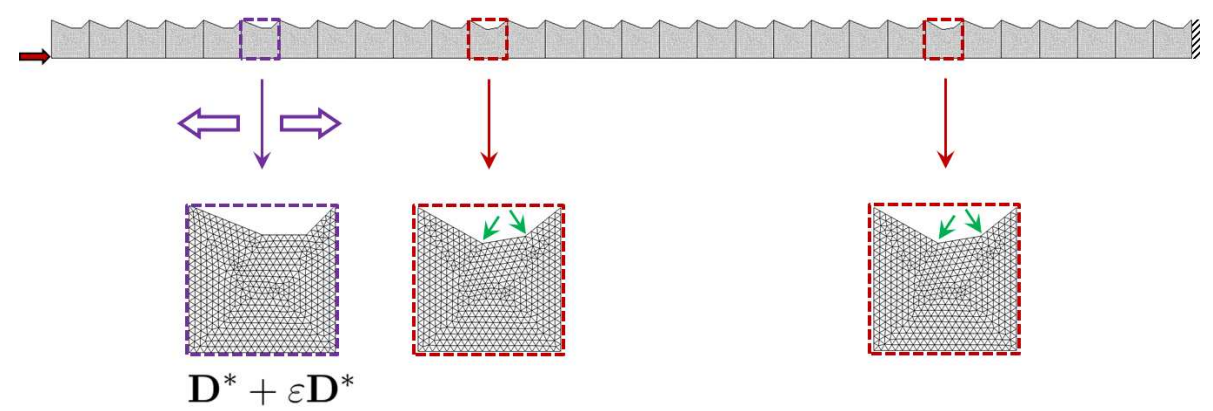

Figure 12: Periodic structure made up of $N=30$ substructures with two controlled perturbed substructures 12 and 24, and containing one uncontrolled perturbed substructure whose location arbitrarily varies.

Again, MC simulations are performed to assess, first, the sensitivity of the FRF of the structure without controlled perturbed substructures, over a frequency band $[5 \mathrm{~Hz}, 2500 \mathrm{~Hz}]$ (see Figure 13). For the sake of clarity, the variation/dispersion of the FRF around the resonance peak at $2110 \mathrm{~Hz}$ is also displayed.

The purpose behind the present analysis is to assess whether the consideration of two controlled substructures (Figure 12), whose DSM is significantly perturbed, 

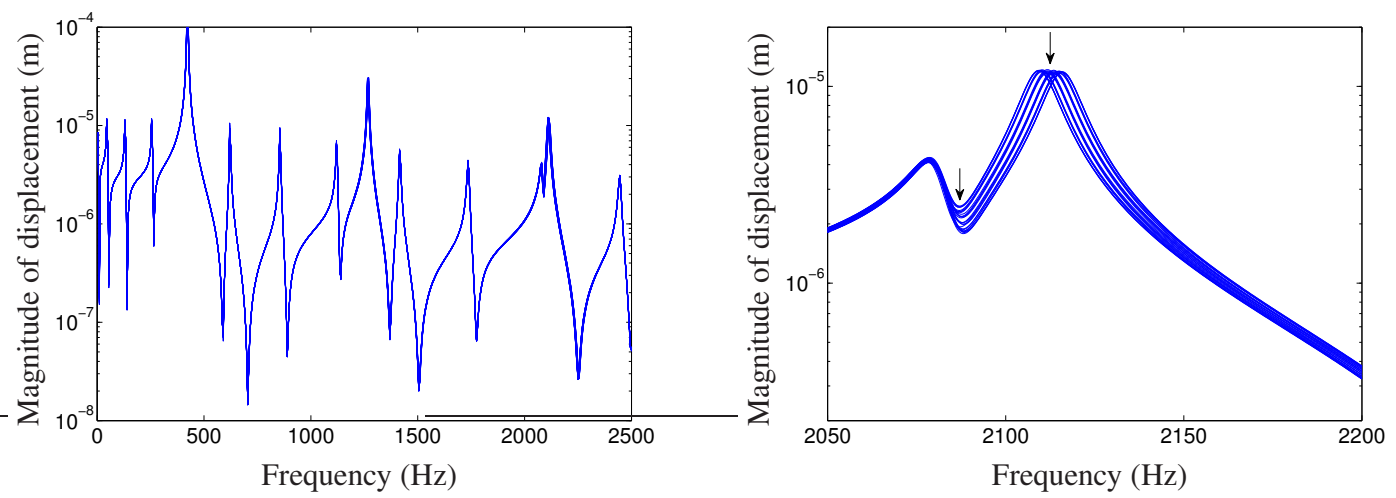

Figure 13: FRF of the periodic structure with one uncontrolled perturbed substructure whose location arbitrarily varies.

enables the dispersion of the FRF to be reduced, especially around the resonance peak at $2110 \mathrm{~Hz}$. A simple trick is considered here which consists in adding those controlled perturbed substructures at the locations where the periodic structure is the most sensitive to the occurrence of an uncontrolled perturbed substructure, i.e., whose DSM is $\mathbf{D}^{*}+\varepsilon \mathbf{D}^{*}$. Such an analysis can be simply achieved in a preprocessing step, by calculating the relative error of the displacement response for each possible location of the uncontrolled perturbed substructure. This yields the locations $p_{1}=12$ and $p_{2}=24$, as shown in Figure 12. Hence, the proposed strategy prevents high sensitivity of the FRF by discarding those possible locations for the uncontrolled perturbed substructure, and considering instead two different controlled perturbed substructures which, in turn, can be subjected to local perturbations, i.e, their DSM can be modified as $0.6 \times\left(\mathbf{D}_{p}^{*}+\varepsilon \mathbf{D}_{p}^{*}\right)$. The dispersion of the FRF of the periodic structure with controlled perturbed substructures $p_{1}=12$ and $p_{2}=24$ is assessed in Figure 14, regarding the occurrence of the uncontrolled perturbation which can be arbitrarily located, outside the perturbed parts but also at the locations of the controlled substructures. As it can be seen, the dispersion of the FRF is significantly reduced around the resonance compared to the case with- 
out controlled perturbations (see Figure 13). As a second advantage of the proposed strategy, the dispersion can be strongly decreased around the anti-resonance at $2080 \mathrm{~Hz}$. As it turns out, these results seem to be very promising and should encourage further investigations on that topic.
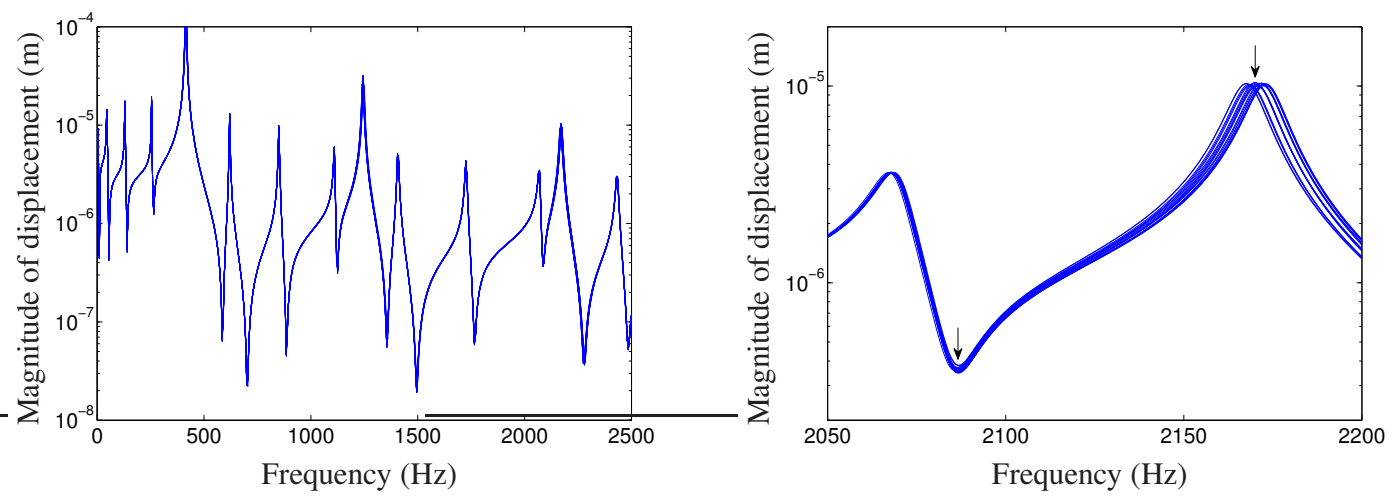

Figure 14: FRF of the periodic structure with two controlled perturbed substructures 12 and 24, and with one uncontrolled perturbed substructure whose location arbitrarily varies.

\section{Concluding remarks}

A WFE-based approach has been proposed to assess the dynamic behavior of periodic structures which are locally perturbed. The remarkable feature of this numerical approach is that it is low time-consuming while keeping the same level of accuracy as the conventional FE method. Within the present study, a model reduction technique has been proposed which involves considering perturbed parts made up of perturbed substructures surrounded by two unperturbed ones. In this framework, a reduced wave-based matrix formulation has been developed through the consideration of small-sized transfer matrices, across the perturbed parts. Numer- 
ical experiments have been carried out which highlight the relevance of the proposed approach in terms of accuracy and computational saving. Also, Monte Carlo simulations have been performed to assess the sensitivity of the FRFs of periodic structures with one or two arbitrarily located perturbed substructures. Additional simulations have been made to examine the feasibility to improve the robustness of periodic structures to the occurrence of arbitrary slight perturbations. The proposed strategy consists in artificially adding several "controlled" perturbations for lowering the sensitivity of the dynamic responses to the occurrence of other uncontrolled perturbations. The analysis has been applied here to time-harmonic elasticity problems but it is clear that the same approach could be used for other time-harmonic linear problems. 


\section{References}

[1] D. Mead, A general theory of harmonic wave propagation in linear periodic systems with multiple coupling, Journal of Sound and Vibration 27 (2) (1973) $235-260$.

[2] W. X. Zhong, F. W. Williams, On the direct solution of wave propagation for repetitive structures, Journal of Sound and Vibration 181 (3) (1995) 485-501.

[3] J.-M. Mencik, M. N. Ichchou, Multi-mode propagation and diffusion in structures through finite elements, European Journal of Mechanics - A/Solids 24 (5) (2005) 877-898.

[4] C. Wilcox, Theory of Bloch waves, Journal d'Analyse Mathématique 33.

[5] J.-M. Mencik, M. N. Ichchou, A substructuring technique for finite element wave propagation in multi-layered systems, Computer Methods in Applied Mechanics and Engineering 197 (6-8) (2008) 505-523.

[6] B. Mace, D. Duhamel, M. Brennan, L. Hinke, Finite element prediction of wave motion in structural waveguides, Journal of the Acoustical Society of America 117 (2005) 2835.

[7] J.-M. Mencik, M. N. Ichchou, Wave finite elements in guided elastodynamics with internal fluid, International Journal of Solids and Structures 44 (2007) $2148-2167$.

[8] E. Manconi, B. Mace, R. Gaziera, Wave finite element analysis of fluid-filled pipes, Proceedings of NOVEM 2009 "Noise and Vibration: Emerging Methods", Oxford, UK. 
[9] W. J. Zhou, M. N. Ichchou, Wave propagation in mechanical waveguide with curved members using wave finite element solution, Computer Methods in Applied Mechanics and Engineering 199 (33-36) (2010) 2099-2109.

[10] L. Gry, C. Gontier, Dynamic modelling of railway track: a periodic model based on a generalized beam formulation, Journal of Sound and Vibration 199 (4) (1997) 531-558.

[11] J. Signorelli, A. von Flotow, Wave propagation, power flow, and resonance in a truss beam, Journal of Sound and Vibration 126 (1) (1988) 127-144.

[12] Y. Waki, B. Mace, M. Brennan, Free and forced vibrations of a tyre using a wave/finite element approach, Journal of Sound and Vibration 323 (3-5) (2009) 737-756.

[13] S. J. Elliott, G. Ni, B. R. Mace, B. Lineton, A wave finite element analysis of the passive cochlea, Journal of the Acoustical Society of America 133 (3) (2013) 1535-1545.

[14] D. Chronopoulos, M. Ichchou, B. Troclet, O. Bareille, Computing the broadband vibroacoustic response of arbitrarily thick layered panels by a wave finite element approach, Applied Acoustics 77 (2014) 89-98.

[15] Q. Serra, M. N. Ichchou, J. F. Deü, Wave properties in poroelastic media using a Wave Finite Element Method, Journal of Sound and Vibration 335 (2015) 125-146.

[16] A. Kessentini, M. Taktak, M. B. Souf, O. Bareille, M. Ichchou, M. Haddar, Computation of the scattering matrix of guided acoustical propagation by the Wave Finite Element approach, Applied Acoustics 108 (2016) 92-100. 
[17] E. Nobrega, F. Gautier, A. Pelat, J. D. Santos, Vibration band gaps for elastic metamaterial rods using wave finite element method, Mechanical Systems and Signal Processing In Press, Corrected Proof, Available online 15 March 2016.

[18] A. Søe-Knudsen, S. Sorokin, On accuracy of the wave finite element predictions of wavenumbers and power flow: A benchmark problem, Journal of Sound and Vibration 330 (12) (2011) 2694-2700.

[19] J.-M. Mencik, On the low- and mid-frequency forced response of elastic systems using wave finite elements with one-dimensional propagation, Computers and Structures 88 (11-12) (2010) 674-689.

[20] D. Duhamel, B. Mace, M. J. Brennan, Finite element analysis of the vibrations of waveguides and periodic structures, Journal of Sound and Vibration 294 (1-2) (2006) 205-220.

[21] Y. Waki, B. Mace, M. Brennan, Numerical issues concerning the wave and finite element method for free and forced vibrations of waveguides, Journal of Sound and Vibration 327 (1-2) (2009) 92-108.

[22] D. Mead, The forced vibration of one-dimensional multi-coupled periodic structures: An application to finite element analysis, Journal of Sound and Vibration 319 (2009) 282-304.

[23] J.-M. Mencik, A wave finite element-based formulation for computing the forced response of structures involving rectangular flat shells, International Journal for Numerical Methods in Engineering 95 (2) (2013) 91-120.

[24] J.-M. Mencik, New advances in the forced response computation of periodic 
structures using the wave finite element (WFE) method, Computational Mechanics 54 (3) (2014) 789-801.

[25] J.-M. Mencik, D. Duhamel, A wave-based model reduction technique for the description of the dynamic behavior of periodic structures involving arbitraryshaped substructures and large-sized finite element models, Finite Elements in Analysis and Design 101 (2015) 1-14.

[26] R. Patel, On computing the eigenvalues of a symplectic pencil, Linear Algebra and its Applications 188-189 (1993) 591-611.

[27] J.-M. Mencik, A model reduction strategy for computing the forced response of elastic waveguides using the wave finite element method, Computer Methods in Applied Mechanics and Engineering 229-232 (2012) 68-86. 